STRUCTURAL SCIENCE CRYSTAL ENGINEERING MATERIALS

ISSN 2052-5206

Received 23 November 2015

Accepted 28 February 2016

Edited by A. J. Blake, University of Nottingham, England

Keywords: Source Function; electron delocalization; chemical transferability; chemical bonding; X-ray derived electron density.

Supporting information: this article has supporting information at journals.iucr.org/b

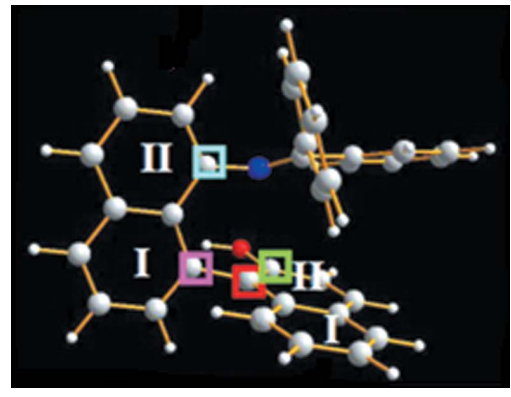

C 2016 International Union of Crystallography

\section{Source Function applied to experimental densities reveals subtle electron-delocalization effects and appraises their transferability properties in crystals}

\author{
Carlo Gatti, ${ }^{a *}$ Gabriele Saleh ${ }^{\mathrm{b} *}$ and Leonardo Lo Presti ${ }^{\mathrm{c}}$
}

${ }^{a}$ CNR-ISTM Istituto di Scienze e Tecnologie Molecolari, via Golgi 19, Milano I-20133, Italy, ${ }^{\mathbf{b}}$ Moscow Institute of Physics and Technology, 9 Institutskiy per, Dolgoprudny, Moscow region 141700, Russian Federation, and ${ }^{\mathbf{c}}$ Dipartimento di Chimica, Università degli Studi di Milano, via Golgi 19, Milano 20133, Italy. *Correspondence e-mail: c.gatti@istm.cnr.it, gabrielesaleh@outlook.com

The Source Function (SF), introduced in 1998 by Richard Bader and Carlo Gatti, is succinctly reviewed and a number of paradigmatic applications to in vacuo and crystal systems are illustrated to exemplify how the SF may be used to discuss chemical bonding in both conventional and highly challenging cases. The SF enables the electron density to be seen at a point determined by source contributions from the atoms or a group of atoms of a system, and it is therefore well linked to the chemist's awareness that any local property and chemical behaviour is to some degree influenced by all the remaining parts of a system. The key and captivating feature of the SF is that its evaluation requires only knowledge of the electron density (ED) of a system, thereby enabling a comparison of $a b$ initio and X-ray diffraction derived electron density properties on a common and rigorous basis. The capability of the SF to detect electrondelocalization effects and to quantify their degree of transferability is systematically explored in this paper through the analysis and comparison of experimentally X-ray derived Source Function patterns in benzene, naphthalene and $( \pm)-8^{\prime}$-benzhydrylideneamino-1,1'-binaphthyl-2-ol (BAB) molecular crystals. It is shown that the SF tool recovers the characteristic SF percentage patterns caused by $\pi$-electron conjugation in the first two paradigmatic aromatic molecules in almost perfect quantitative agreement with those obtained from $a b$ initio periodic calculations. Moreover, the effect of chemical substitution on the degree of transferability of such patterns to the benzene- and naphthalene-like moieties of BAB is neatly shown and quantified by the observed systematic deviations, relative to benzene and naphthalene, of only those SF contributions from the substituted C atoms. Finally, the capability of the SF to reveal electrondelocalization effects is challenged by using a promolecule density, rather than the proper quantum mechanical density, to determine the changes in SF patterns along the cyclohexene, 1,3-cyclohexadiene and benzene molecule series. It is shown that, differently from the proper quantum density, the promolecular density is unable to reproduce the SF trends anticipated by the increase of electron delocalization along the series, therefore ruling out the geometrical effect as being the only cause for the observed SF patterns changes.

\section{Looking at the electron density from a new} perspective

This feature article deals with applications of the Source Function (SF) descriptor (Bader \& Gatti, 1998) to electron densities (EDs) derived from X-ray diffraction data as a means to reveal electron-delocalization effects in crystals. The possibility of using the SF to detect such effects has already been firmly assessed for isolated molecules and for EDs from first-principles computations (Gatti, 2012; Monza et al., 2011; Gatti et al., 2016), but extending to crystals and experimental 
EDs, although being reported at a number of conferences (Gatti et al., 2012; Gatti, 2013a), in a PhD thesis (Saleh, 2014) and in two papers discussing heteroaromaticity in a benzothiazol-substituted phosphane (Hey et al., 2013) or antiaromaticity in cyclopentadienone derivatives (Pal et al., 2014), still needs to be fully demonstrated in the literature. Obvious, yet quite interesting and still unanswered questions are whether the EDs from X-ray data may be accurate enough to reveal the subtle features caused by electron pairing and whether these same features are not only detectable, but also reproducible and transferable, whenever appropriate.

Before coming to the main subject of this work in $\S 2$, the Source Function tool is briefly reviewed and, to help the reader, a few examples of its use in chemical bonding of increasing complexity, in vacuo and in crystals, are also illustrated in the paragraph. A comprehensive discussion on the Source Function tool and its applications may be found in Gatti (2012) and, at a more narrative and less analytical level, in Gatti (2013b).

\subsection{Source Function for electron density}

In a nutshell, the SF enables the properties of the electron density $\rho$, at any point $\mathbf{r}$ in $R^{3}$, in terms of source contributions from all other points $\mathbf{r}^{\prime}$ and within an interesting cause-effect relationship to be studied (Bader \& Gatti, 1998; Gatti, 2012). The cause for the effect, i.e. the value of the ED at $\mathbf{r}$, is related to the local behaviour, in terms of the Laplacian, $\nabla^{2} \rho$, of this same scalar $\rho$ at all other points of space $\mathbf{r}^{\prime}$. On these grounds it is evident that the SF is an interpretive tool which is deeply tied to one of the main operative notions of chemistry, namely that any local property and chemical behaviour of a system is to some extent always influenced by the remaining parts of the system. Whether such an influence is small or large, it is just quantified through the SF.

Herein we examine all this in more detail. More than 20 years ago, Richard Bader and one of us (CG) were having a glance through chapter I of Morse \& Feshbach's (1981) book on Methods of Theoretical Physics when we were suddenly inspired by the section on 'A solution of Poisson's Equation'. We realised that Poisson's equation could be solved through a potential given by the electron density itself and that the electron density at a point $\mathbf{r}$ could then be seen as determined by contributions from a local source $\operatorname{LS}\left(\mathbf{r}, \mathbf{r}^{\prime}\right)$, operating at all other points $\mathbf{r}^{\prime}$ in the space

$$
\rho(\mathbf{r})=\int \operatorname{LS}\left(\mathbf{r}, \mathbf{r}^{\prime}\right) \mathrm{d} \mathbf{r}^{\prime},
$$

and given in terms of the Laplacian of the electron density

$$
\operatorname{LS}\left(\mathbf{r}, \mathbf{r}^{\prime}\right)=-\left(4 \pi \cdot\left|\mathbf{r}-\mathbf{r}^{\prime}\right|\right)^{-1} \cdot \nabla^{2} \rho\left(\mathbf{r}^{\prime}\right) .
$$

In equation (2) $\left(4 \pi \cdot \mid \mathbf{r}-\mathbf{r}^{\prime}\right)^{-1}$ is Green's function or an influence function (Arfken, 1985), expressing the influence or effectiveness of $\nabla^{2} \rho\left(\mathbf{r}^{\prime}\right) \mathrm{d} \mathbf{r}^{\prime}$ in contributing to the effect $\rho(\mathbf{r})$. The operation of the local source over the whole space [equation (1)] may then be replaced [equation (3)] with separate LS integrations over atomic basins $\Omega$ defined by the
Quantum Theory of Atoms in Molecules (QTAIM; Bader, 1990), i.e. over the disjoint and exhaustive regions of space bounded by zero-flux surfaces in the $\nabla \rho(\mathbf{r})$ vector field

$$
\rho(\mathbf{r})=\int \operatorname{LS}\left(\mathbf{r}, \mathbf{r}^{\prime}\right) \mathrm{d} \mathbf{r}^{\prime}=\sum_{\Omega} \int_{\Omega} \operatorname{LS}\left(\mathbf{r}, \mathbf{r}^{\prime}\right) \mathrm{d} \mathbf{r}^{\prime}=\sum_{\Omega} \operatorname{SF}(\mathbf{r}, \Omega) .
$$

In such a way, $\rho(\mathbf{r})$ is seen as determined by the sum of atomic contributions $\operatorname{SF}(\mathbf{r} ; \Omega)$, each of which is termed the Source Function from the atom $\Omega$ to the ED at a reference point (hereinafter, $r p$ ) r. Any other scheme could be clearly chosen to partition the LS integration over $R^{3}$ into separate contributions. However, adopting the QTAIM recipe has the great advantage of ensuring an association of the $\operatorname{SF}(\mathbf{r}, \Omega)$ sources to contributions from atoms or groups of atoms rigorously defined through quantum mechanics (Bader, 1990). Another interesting feature of the SF tool deserves to be emphasized. Equations (1)-(3) clearly show that the $\operatorname{SF}(\mathbf{r} ; \Omega)$ values are amenable to experimental determination, provided an accurate $\nabla^{2} \rho$ distribution, like that derived from highquality single-crystal X-ray diffraction intensity data and using the so-called multipole models (Hansen \& Coppens, 1978; Stewart et al., 1975), is available. The SF tool may thus provide a privileged bridge between theory and experiment, as it allows the comparison of both outcomes on the same grounds (Gatti, 2012, 2013a,b; Lo Presti \& Gatti, 2009). The same holds true for the electrostatic potential $V_{\text {elec }}$, which bears a formal analogy with the SF analysis as both $V_{\text {elec }}$ and $\rho(\mathbf{r})$ are possible solutions of Poisson's equation. By combining equations (1) and (2), it is evident that $\rho(\mathbf{r})$ is given by an expression that mimics that for $V_{\text {elec }}$ at $\mathbf{r}$. The ED may be envisaged as the potential generated by its Laplacian distribution (Bader \& Gatti, 1998), in full accordance with the physical interpretation of $\nabla^{2} \rho\left(\mathbf{r}^{\prime}\right)$ as the cause of $\rho(\mathbf{r})$. Likewise, it is obvious that $V_{\text {elec }}(\mathbf{r})$ may also be decomposed in atomic contributions, as it is for $\rho(\mathbf{r})$. The analogy between $V_{\text {elec }}$ and $\rho(\mathbf{r})$ reconstructions, in terms of the ED and the ED Laplacian distributions, further attests to the fact that the $\operatorname{SF}(\mathbf{r}, \Omega)$ atomic contributions in equation (3) do not represent a direct $\mathrm{ED}$ donation from these atoms to $\rho(\mathbf{r})$, but simply their own capability to influence or determine such density, similar to the role the ED itself has in determining $V_{\text {elec }}$.

The SF does not merely tell us that the ED is only apparently a local quantity. This piece of information is already well known from Density Functional Theory (DFT), which states that the electron density at any point is a unique function of the position and nuclear charge of all nuclei in the system. The $\mathrm{SF}$, in fact, conveys additional and valuable detail. It quantifies and translates such non-locality in terms of the language of chemistry, as each $\operatorname{SF}(\mathbf{r} ; \Omega)$ represents a measure of how an atom $\Omega$, or a group of atoms $\Omega$, contributes to determine the density at $\mathbf{r}$, relative to contributions from other atoms or groups of atoms in the system. In particular, the SF enables us to see the local response of the density to any perturbation, like a change in substituent or in the environment of a system, in terms of how the various moieties of the system (atoms, 
groups of atoms, atoms of the environment etc.) contribute to determine such a response. Due to these interesting properties, the SF descriptor has been extensively applied to discuss bonding features in highly debated cases. These include hydrogen bonds, multi-center bonds, metal-metal and metalligand bonds, both in molecules and crystals and using information from theoretical or experimental densities (Gatti et al., 2003; Farrugia et al., 2006; Gatti \& Lasi, 2007; Farrugia et al., 2009; McGrady et al., 2009; Lo Presti et al., 2011; Gatti, 2012; Schmökel et al., 2012; Engels et al., 2012; Gatti, 2013a,b; Saleh et al., 2013). A few paradigmatic cases are briefly illustrated here as a useful premise to $\$ 2$ where the capability of the SF to reveal electron-delocalization effects from the ED alone and independently from any molecular orbital scheme or decomposition will be first reviewed and then extended to the ED from experiment.

1.1.1. The Source Function for the electron density and chemical bonding. The study of bonding through the SF implies the choice of a suitable $r p$, where selecting the bond critical point (b.c.p.) represents the usual and least-biased assumption. The b.c.p. is a point where $\nabla \rho$ vanishes and where $\rho$ attains its minimum value along the bond path - the line of maximum ED relative to any lateral displacement linking two bonded nuclei, according to the QTAIM criterion (Bader, 1990). The reference point choices other than b.c.p.s are, however, clearly possible (Gatti, 2012) and often appropriate (see below). SF contributions may either be compared as absolute values or in terms of percentage values $\mathrm{SF} \%(r p, \Omega)$

$$
\mathrm{SF} \%(r p, \Omega)=\frac{\mathrm{SF}(r p, \Omega)}{\rho(r p)} \times 100
$$

expressing the relative ability of an $\Omega$ atom to determine $\rho$ at the $r p$.

In the series ethane, ethene, ethyne, the $\mathrm{C}$ atoms dominate the ED reconstruction at the carbon-carbon b.c.p. (Gatti \& Lasi, 2007). The cumulative SF\% contribution from the $\mathrm{C}$ atoms reaches a value of $96 \%$ in the case of ethyne and it is found to increase, from a value of $79 \%$ in ethane, with the formal $\mathrm{CC}$ bond order increase along the series (Fig. 1, first row). This result mirrors the prospect that the more covalently bonded the two atoms, the higher will be their ability to contribute to the ED value at their intervening b.c.p. and, thus, their related SF\% contribution (Gatti et al., 2003). When the interactions are less localized, the SF contributions are anticipated to become more delocalized over the various molecular moieties and the SF\% to become generally smaller in value (Gatti et al., 2003; Gatti \& Lasi, 2007). This is illustrated for two members ( $M=\mathrm{Pd}$ and $\mathrm{Mo})$ of the $M_{2}$ (formamidinate $)_{4}(M=\mathrm{Nb}, \mathrm{Mo}, \mathrm{Tc}, \mathrm{Ru}, \mathrm{Rh}$ and $\mathrm{Pd})$ binuclear $4 d$ metal complex series (Fig. 1, second row). These isostructural compounds differ in the nature of their metal atom and in the related number of electrons available for the metal-metal bond, yielding formal bond orders ranging from zero $(M=\mathrm{Pd})$ to four $(M=\mathrm{Mo})$. A bond path is found to link the metal atoms in all complexes, including that of $\mathrm{Pd}$ with zero formal bond order. However, the SF\% values reconstructing the ED at the metal-metal b.c.p. clearly distinguish the different nature of bonding in the various complexes (Gatti \& Lasi, 2007). When $M=$ Mo the two metal atoms contribute to determine more than $81 \%$ of the b.c.p. ED value, while for $M=\mathrm{Pd}$ it is the four formamidinate ligands which dominate such a value, with an overall $\mathrm{SF} \%$ value equal to $66 \%$ (16.5\% for each ligand, see Fig. 1, second row).

The next two examples in the third and fourth row of Fig. 1 concern joint experimental and theoretical ED investigations of bonding in molecular crystals. The former example, by Farrugia et al. (2006), concerns bonding between a delocalized $\pi$-hydrocarbyl system and a transition metal atom in the trimethylenemethane (TMM) complex $\left(\mathrm{Fe}\left(\eta^{4}-\mathrm{C}_{\left.\left\{\mathrm{CH}_{2}\right\}_{3}\right)-}\right.\right.$ $\left.(\mathrm{CO})_{3}\right)$, while the second, by McGrady et al. (2009), relates to the metal-ligand bonding in the metal-silane $\sigma$-complex $\left[\mathrm{Cp}^{\prime} \mathrm{Mn}(\mathrm{CO})_{2}\left(\eta^{2}-\mathrm{HSiFPh}_{2}\right)\right]\left(\mathrm{Cp}^{\prime}=\eta^{5}-\mathrm{C}_{5} \mathrm{H}_{4} \mathrm{Me}\right)$. In both cases,

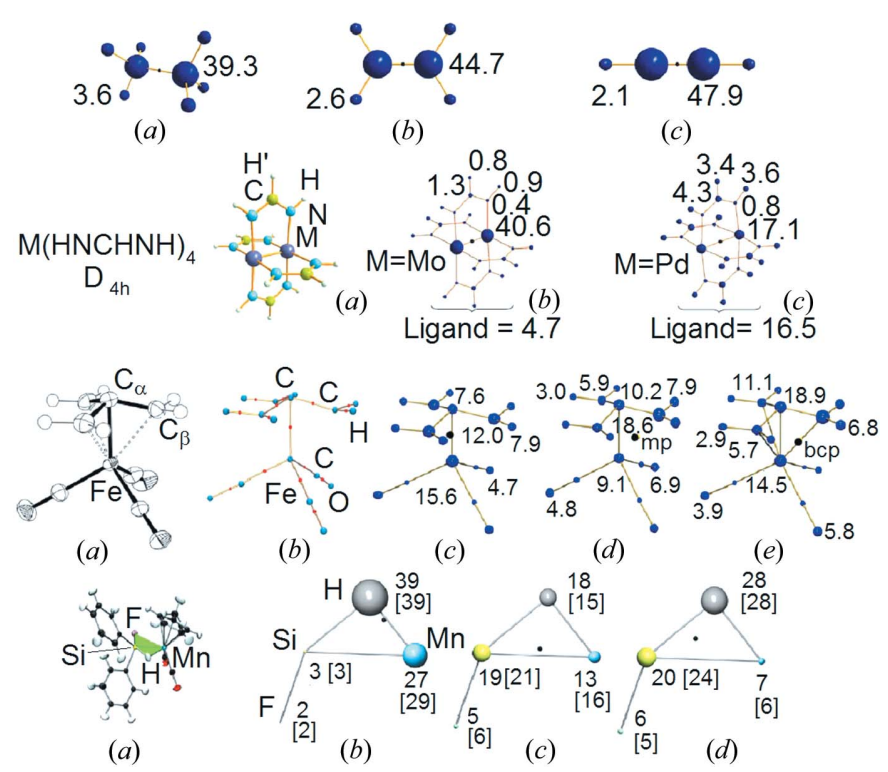

Figure 1

Source Function analysis of conventional and less conventional bonding interactions in vacuo and in the solid state. The reported numbers in the figure refer to atomic or group Source Function percentage values, SF\%, relative to a given reference point, $r p$ (denoted by a black dot in the various $\mathrm{SF} \%$ panels of the figure). SF\% values are also displayed as spheres whose volume is proportional to the values themselves. First row: (a) ethane, $(b)$ ethene and (c) ethyne. The reference point, $r p$ is the CC b.c.p. (modified from Gatti \& Lasi, 2007, with permission). Second row: (a) graphical representation of the $M_{2}$ (formamidinate) (fisostructural $_{4}$ binuclear metal complexes. SF\% values ( $r p=M-M$ b.c.p.) for ( $b$ ) $M=$ Mo and (c) $M=$ Pd complexes (adapted from Gatti \& Lasi, 2007, with permission). Third row: Trimethylenemethane (TMM) complex $\left(\mathrm{Fe}\left(\eta^{4}-\right.\right.$ $\left.\left.\mathrm{C}\left\{\mathrm{CH}_{2}\right\}_{3}\right)-(\mathrm{CO})_{3}\right):(a)$ ORTEP diagram; (b) molecular graph, bond paths and b.c.p.s (red dots) at an equilibrium distance; $(c)-(e) \mathrm{SF} \%$ values for rps placed at (c) the $\mathrm{Fe}-\mathrm{C}_{\alpha}$ b.c.p., $(d)$ the $\mathrm{Fe}-\mathrm{C}_{\beta}$ mid-point (mp) and (e) the $\mathrm{Fe}-\mathrm{C}_{\beta}$ b.c.p. for a slightly deformed geometry of the complex, where three bond paths linking the iron nucleus to the $\mathrm{C}_{\beta} \mathrm{C}$ atom nuclei also occur (adapted from Farrugia et al., 2006 with permission from American Chemical Society; Copyright 2006). Fourth row: Nature of bonding in the metal-silane $\sigma$-complex $\left[\mathrm{Cp}^{\prime} \mathrm{Mn}(\mathrm{CO})_{2}\left(\eta^{2}-\mathrm{HSiFPh}_{2}\right)\right]\left(\mathrm{Cp}^{\prime}=\eta^{5}-\mathrm{C}_{5} \mathrm{H}_{4} \mathrm{Me}\right)$ : (a) molecular structure; $(b)-(d) \mathrm{SF} \%$ values for $r p s$ placed at $(b)$ the $\mathrm{Mn}-\mathrm{H}$ b.c.p., (c) the $\mathrm{Mn}-\mathrm{Si}$ b.c.p. and $(d)$ the $\mathrm{Si}-\mathrm{H}$ b.c.p. The reported values are derived from the experimental and $a b$ initio (in parenthesis) electron density distributions (adapted from McGrady et al., 2009, with permission from American Chemical Society; Copyright 2009). 
the fairly delocalized and non-classical character of interactions makes the SF quite an attractive tool for their study. Transition metal $\pi$-hydrocarbyl complexes are characterized by high fluxional mobility of the ligands, challenging the twocenter view of bonding tied to the bond path criterion. Furthermore, due to the typical flatness of the potential energy surfaces and of the electron distributions of metal- $(\pi-$ hydrocarbyl) interactions, fewer $M-\mathrm{C}$ bond paths than the formal hapticity of the complex would anticipate are normally observed (Farrugia et al., 2006). Indeed, only one bond path, linking the $\mathrm{C}_{\alpha}$ atom to the iron was recovered in both the experimental and theoretical ED topologies, while no such paths were found between the metal and any of the three $\mathrm{C}_{\beta}$ atoms, implying a description of the system, according to the bond path criterion, as of an $\eta^{1}$ complex.

However, other theoretical and experimental evidence, like the normal-mode analysis, the portraits of the Kohn-Sham orbitals, the ESCA and photoelectron spectra and the NMR barrier to rotation of the TMM ligand in the complex, all seem in favour of a noteworthy interaction of the iron with the whole $\pi$-electron system. As a consequence, $\mathrm{C}_{\beta}$ atoms also appear to be involved in bonding with the metal and the system should therefore be classified as an $\eta^{4}$ complex. The SF analysis neatly confirms such a view. When the $r p$ is placed at the $\mathrm{Fe}-\mathrm{C}_{\alpha}$ b.c.p., the $\mathrm{SF} \%$ contributions from the TMM ligand are fairly delocalized and the contribution from $\mathrm{C}_{\beta}$ to the $\mathrm{Fe}-\mathrm{C}_{\alpha}$ b.c.p. density is even larger than that from $\mathrm{C}_{\alpha}$, despite the lack of a bond path between the iron and the $\mathrm{C}_{\beta}$ atoms, and despite the fact that these percentage sources refer to the density reconstruction at a b.c.p. involving $\mathrm{C}_{\alpha}$ rather than $\mathrm{C}_{\beta}$ (Fig. 1, third row, $c$ ). Larger sources from $\mathrm{C}_{\beta}$ than from $\mathrm{C}_{\alpha}$ are then found, a fortiori, if the $\mathrm{Fe}-\mathrm{C}_{\beta}$ axis mid-point is taken as an $r p$, the latter representing the least biased choice of an $r p$ for the interaction between two atoms when a b.c.p. is lacking (Fig. 1, third row, $d$ ). At both mentioned rps, the three methylene groups determine about $60 \%$ of these rps $\mathrm{ED}$ values, the contribution from the central $\mathrm{C}_{\alpha}$ atom being, instead, as small as $7-10 \%$.

It is worth noting that such a delocalized picture of the iron( $\pi$-hydrocarbyl) interaction is fully confirmed by resorting to the $(\mathrm{Fe}, \mathrm{C})$ delocalization indices, whose evaluation requires knowledge of the pair density of the system $\pi\left(\mathbf{r}_{1}, \mathbf{r}_{2}\right)$, or, at least, of the first-order density matrix, in the approximation of a single-determinant wavefunction (Gatti \& Macchi, 2012). The delocalization index, $\delta\left(\Omega_{i}, \Omega_{j}\right)$, expresses the number of electron pairs which are exchanged (shared) between two atoms $\Omega_{i}$ and $\Omega_{j}$, and is thus a physical measure of their bonding interaction (Fradera et al., 1999). Analogously to the $\mathrm{SF}$ tool, such a measure also does not necessitate that the two involved atoms be linked through a bond path. The $\delta\left(\mathrm{Fe}, \mathrm{C}_{\beta}\right)$ value of 0.571 is not only far from being marginal, but even $\sim 40 \%$ larger than the $\delta\left(\mathrm{Fe}, \mathrm{C}_{\alpha}\right)$ value of 0.369 , despite $\mathrm{C}_{\alpha}$ being closer than $\mathrm{C}_{\beta}$ to the $\mathrm{Fe}$ atom $\left[R_{\mathrm{e}}\left(\mathrm{Fe}-\mathrm{C}_{\alpha}\right)=1.945 \AA ; R_{\mathrm{e}}\right.$ $\left.\left(\mathrm{Fe}-\mathrm{C}_{\beta}\right)=2.12-2.13 \AA\right]$.

One more observation supports the view of $\left(\mathrm{Fe}\left(\eta^{4}-\right.\right.$ $\left.\left.\mathrm{C}\left\{\mathrm{CH}_{2}\right\}_{3}\right)-(\mathrm{CO})_{3}\right)$ as an $\eta^{4}$ complex. Both experimental and in vacuo ab initio geometries of the complex occur close to structural catastrophe points, so that one anticipates that new structures might result even for very small atomic displacements from such nuclear configurations. Indeed, when the $\mathrm{Fe}-\mathrm{C}_{\alpha}-\mathrm{C}_{\beta}$ angle is decreased by just $3^{\circ}$, while keeping the original $C_{3 v}$ symmetry of the in vacuo ab initio geometry, three more bond paths between the $\mathrm{Fe}$ atom and the $\mathrm{C}_{\beta}$ atoms emerge, in accordance with a $\eta^{4}$ complex view also in terms of the bond-path structural criterion (Farrugia et al., 2006).

Interestingly, the $\mathrm{SF}$ reconstruction pattern for the $\mathrm{Fe}-\mathrm{C}_{\beta}$ b.c.p. density of the slightly distorted structure hardly changes relative to that for the $\mathrm{Fe}-\mathrm{C}_{\beta}$ mid-point in the minimum energy structure (compare $d$ and $e$, third row, Fig. 1), and the corresponding delocalization indices also behave similarly. It is clear that defining a structure through ED topology and close to catastrophe points is by its own nature a discontinuous process, while using the SF analysis for such a purpose leads to more stable and chemically reasonable structural views (Ponec \& Gatti, 2009; Gatti, 2013b). More importantly, these views turn out to be in agreement with most of the available experimental or theoretical evidence (see earlier).

In summary, the study of this complex reveals that the SF analysis is able to mimic the picture of bonding provided by an elaborate tool like the delocalization index, which requires knowledge of at least the full first-order density matrix, rather than that of its diagonal elements only. Though rooted on an empirical basis, the observation of an existing relationship between the two tools could be made in several circumstances (Gatti \& Lasi, 2007; Gatti, 2012).

The last example shown in Fig. 1 illustrates another interesting case where a multicenter picture of bonding is required or, in other words, where non-local effects on bonding play a relevant role. Silane $\sigma$-bond complexes are the second largest class of $\sigma$-bond complexes after molecular hydrogen systems, and also serve as a model for $\mathrm{C}-\mathrm{H}$ activation by a transition metal center. McGrady et al. (2009) have focused their study on a series of complexes [Cp $\left.\mathrm{Cn}(\mathrm{CO})_{2}\left(\eta^{2}-\mathrm{HSi} X Y_{2}\right)\right]$, with $X=$ $\mathrm{H}, \mathrm{F}$ and $\mathrm{Cl}$, respectively, and $Y$ being $\mathrm{Ph}$ for $X=\mathrm{H}, \mathrm{F}$ or $\mathrm{Cl}$ for $X=\mathrm{Cl}$. They found that the $\mathrm{Mn}\left(\eta^{2}-\mathrm{SiH}\right)$ bonding in all of these systems occurs through an asymmetric oxidative addition reaction coordinate. The $\mathrm{Mn}-\mathrm{H}$ bond is formed at an early stage, while $\mathrm{Mn}-\mathrm{Si}$ bonding turns out to be guided and enforced by the extent of $\mathrm{Mn} \rightarrow \sigma^{*}(X-\mathrm{Si}-\mathrm{H}) \pi$-back donation. By displacing electrons into a three-center ligand orbital with $\mathrm{Si}-X$ and $\mathrm{Si}-\mathrm{H}$ antibonding character, such a back-donation simultaneously activates both the $\eta^{2}$-coordinating $\mathrm{Si}-\mathrm{H}$ bond and the $\mathrm{Si}-X$ bond in the trans position. The larger the electron-withdrawing character of $X$, the greater the $\mathrm{Si}-X$ and $\mathrm{Si}-\mathrm{H}$ bond activation due to the enhanced $\mathrm{Mn} \rightarrow$ ligand $\pi$-back donation. The SF tool, applied to both the experimental and theoretical charge densities of the three investigated complexes, could provide real space evidence of the illustrated Molecular Orbital model interpretation, which is clearly feasible only on a theoretical basis.

The structure for $X=\mathrm{F}$ is shown in Fig. 1, fourth row, panel $(a)$, while panels $(b)-(d)$ show how the various atoms contribute to determine the EDs at the b.c.p.s of the $\operatorname{Mn}\left(\eta^{2}\right.$ $\mathrm{HSiFPh}_{2}$ ) three-membered ring moiety. As anticipated for a 
strongly localized $\mathrm{Mn}-\mathrm{H}$ bond, the $\mathrm{SF} \%$ contributions from the $\mathrm{Mn}(27 \% ; 29 \%$, experimental values in italic) and the $\mathrm{H}$ $(39 \% ; 39 \%)$ atoms dominate the ED at the $\mathrm{Mn}-\mathrm{H}$ b.c.p., while the $\mathrm{Si}$ atom provides only a marginal source for such a density (SF\%: 3\%; 3\%). The other two studied complexes behave similarly, corroborating the view of the oxidative addition of the silane ligand to $\mathrm{Mn}$ as an asymmetric process and one where the $\mathrm{Mn}-\mathrm{H}$ bond forms at an early stage and is barely influenced by the extent of the $\mathrm{Mn} \rightarrow \sigma^{*}(X-\mathrm{Si}-\mathrm{H}) \pi$ back donation. The scenario radically changes when the $r p$ is moved to the $\mathrm{Mn}-\mathrm{Si}$ b.c.p. as, here, all the three atoms of the ring are found to contribute to a very similar extent to the ED reconstruction $(13 \%, 19 \%, 18 \% ; 16 \%, 21 \%, 15 \%$, for $\mathrm{Mn}, \mathrm{Si}$ and $\mathrm{H}$, respectively; experimental values in italic). These largely delocalized sources are just mirroring a delocalized bonding interaction, where the formation of the $\mathrm{Mn}-\mathrm{Si}$ bond affects the $\mathrm{Si}-\mathrm{H}$ bond, thus increasing the capability of the $\mathrm{H}$ to contribute to the $\mathrm{Mn}-\mathrm{Si}$ b.c.p. density because of the $\pi$ back donation from the metal into the antibonding $\sigma^{*}(X-$ $\mathrm{Si}-\mathrm{H})$ orbital. Very delocalized sources are likewise observed for the $\mathrm{ED}$ reconstruction at the $\mathrm{Si}-\mathrm{H}$ b.c.p., with a nonnegligible contribution from the $\mathrm{Mn}$ atom (SF\%: 7\%; 6\%). In summary, the SF tool, being defined in terms of an observable, provides a rigorous validation of the $M O$ interpretation of the $\mathrm{Mn}\left(\eta^{2}-\mathrm{SiH}\right)$ interaction, one where $\mathrm{Si}-\mathrm{Mn}$ bonding dictates its strength as a result of a complex interplay of contributions from all four atoms of the $\mathrm{Mn}\left(\eta^{2}-\mathrm{HSi} X\right)$ moiety (Fig. 1, fourth row, $d$ ).

\section{Detecting electron-delocalization effects through the source function for the electron density}

Examples shown in the previous section demonstrate that the SF tool is able to detect the contribution to a bond from atoms other than those directly bonded. The interesting question arises as to whether the SF, despite being based on quantities derived from the ED only, is also able to reveal electron conjugation/delocalization effects, like those, for instance, leading to aromaticity. In essence: if electron delocalization takes place between atoms (or groups of atoms) $A$ and $B$, does this have any influence on how atom (or group of atoms) $A$ determines the ED at the various points of $B$ and vice versa? Answers to such questions were given by Gatti (2012), Monza et al. (2011) and Gatti et al. (2016) using ab initio in vacuo ED. A brief summary of the tenets and outcomes of these works is given in $\$ 2.1$, while extension to crystals and experimentally derived ED is presented in $\$ \$ 2.3$ and 2.4. $\$ 2.2$ deals instead with the discussion of a number of criticisms about the effective capability of the SF tool to detect electron-delocalization effects.

Here we mention a few general remarks concerning the methods customarily used to reveal electron-delocalization effects and the motivations which suggest also adopting the SF analysis as a precious tool in such an arena.

In spite of their ubiquitous use in chemistry and being cornerstones of chemical understanding and classification, electron delocalization and aromaticity measures cannot be directly associated with quantum-mechanical observations and are thus not amenable to a rigorous definition (Poater et al., 2005; Bultinck, 2007). They affect, however, the structural, thermochemical, spectroscopic and magnetic properties of a system - all these properties being clearly interrelated as all depend on the system's electronic structure and distribution (for comprehensive and updated bibliography see Poater et al., 2005; Feixas et al., 2015; Cocq et al., 2015, and the references therein). The use of such distributions and b.c.p. properties to discuss electron conjugation, hyperconjugation, aromaticity and homoaromaticity was pioneered by Bader et al. (1983) and Cremer et al. (1983) in two seminal papers which headed the exploitation of the electron-based descriptors to shed light on electron-delocalization phenomena. Electronic effects predicted by orbital models were shown to be translated into observable properties of the ED distribution, with the advantage that being based on an observable these properties may equally be studied in non-planar systems, where the $\sigma-\pi$ separation of the molecular orbital models is no longer feasible. Descriptors nowadays are very well known; like the ED-based bond orders, the bond ellipticity and the degree of alignment of the axes defining the plane of $\pi$-electron distribution of consecutively joined $\mathrm{C}-\mathrm{C}$ bonds had then been introduced, along with several successful examples of the application of the method to both planar and non-planar conjugated compounds. However, despite the ED showing evident, though indirect, marks of the electron-delocalization effects, their true origin and mechanism lie in the quantummechanical correlated motion of electron pairs. Its description is fully accounted for by the pair density and by the so-called exchange-correlation density, $\rho_{2, x c}\left(\mathbf{r}_{1}, \mathbf{r}_{2}\right)$, derived thereof (Poater et al., 2005). The latter measures the deviation between the true pair density of a system and that given by the purely classical description of a product of two independent electron densities, and it is thus no surprise that several electron-delocalization descriptors, including the delocalization indices $\delta$ mentioned earlier, occur, all defined through $\rho_{2, x c}$. These tools have progressively replaced those based on the ED alone to discuss electron conjugation effects and are now being extensively applied to systems in vacuo. In particular, presently the most popular aromaticity measures, like the multi-center index (MCI; Bultinck et al., 2006), the Fermi hole delocalization density index (FHDD; Matta \& HernándezTrujillo, 2003), the para-delocalization index (PDI; Poater et al., 2003), the FLUctation index (FLU; Matito et al., 2005), are all defined in terms of suitably selected and weighted combinations of $\delta\left(\Omega_{i}, \Omega_{j}\right)$ values.

So, given these premises, why should one make use of the ED-based SF approach in such an area? As already discussed in $\$ 1$, the first obvious and great advantage of the SF, relative to $\delta$, is that of being defined in terms of quantities which are easily derived both from charge-density quality X-ray diffraction works and $a b$ initio studies, and irrespective of the state of the matter (pair densities are, indeed, generally not implemented in periodic wavefunctions codes). Secondly, the SF approach, along with that based on delocalization indices, 
shares the interesting feature of providing a measure of the electronic connection between two different, possibly distant and potentially interacting regions. Connections of this kind are profoundly tied to electron-delocalization mechanisms, with those based on $\delta$ symmetrically relating the action of two atoms or groups of atoms $\Omega_{1}$ and $\Omega_{2}$, while those based on the $\mathrm{SF}$ approach establishing an asymmetric cause-effect link between an atom or a group of atoms and a reference point, usually associated with a bond. The two approaches may thus be characterized in terms of atom(s)-atom(s) and atom(s)bond electron-delocalization relationships, respectively. Besides, the freedom in selection of the $r p$ enhances the chemical insight that the SF approach may convey (Monza et al., 2011; Gatti, 2012). By varying the $r p$, the change of role any given atom has on determining the ED of different bonding regions may be easily evaluated, thus bringing to the fore which of these regions are more responsive to electron-delocalization effects due to that atom (see below).

\subsection{In vacuo systems and ab initio electron densities}

First attempts to reveal electron-delocalization effects through the SF concerned archetypal, planar $\pi$-conjugated organic molecules in vacuo, such as benzene, biphenyl, polycyclic aromatic hydrocarbons (PAH) and differently unsaturated cyclic/polycyclic hydrocarbons (Monza et al., 2011; Gatti, 2012). An extension of such an analysis to non-planar aromatic and homoaromatic molecules was also very recently successfully pursued and it is currently in the press (Gatti et al., 2016).

The guiding concept behind all these studies was to verify whether the presence of electron delocalization is reflected in an increased ability to determine the ED along a given bond by the distant, although through-bonds connected, atomic basins and, at the same time, in a decreased ability to do so by the two atoms directly involved in the bond. Such an adjustment of sources should then translate into a pictorial pattern of enhanced and reduced atomic SF contributions from, respectively, distant and nearby atoms compared with the case of a partially or fully saturated network of bonds.

As an illustration, the very simple, yet paradigmatic case of the cyclohexene, 1,3-cyclohexadiene and benzene sequence of molecules (Gatti, 2012; Monza et al., 2011) is reviewed here. Electron-delocalization effects are expected to increase along this series and the b.c.p. of the bond with the largest doublebond character in each system (C1-C6 in Fig. 2) may be initially selected as a viable $r p$. It may yet be envisaged that such increasing electron-delocalization effects are not directly visible through the $\mathrm{SF}$ when the $r p$ is taken at a point lying in the nodal plane of the $\pi$-orbitals, and so at a point where the $\mathrm{ED}$ of the $\pi$-orbitals vanishes. Indeed, it had been previously pointed out, though without proof, that ' $\pi$-electron delocalization in the benzene ring is not manifest in the SF when the $r p$ is taken at the CC b.c.p.' (Farrugia \& Macchi, 2009).

However, since $\sigma$ - and $\pi$-distributions are self-consistently interrelated, rather than being independent from one another, Gatti (2012) and Monza et al. (2011) speculated that some, albeit a small effect of electron delocalization might also be visible when the $r p$ lies in the $\pi$-nodal plane, even though $\pi$ orbitals do not directly contribute to the electron density in that plane. Results shown in the first row of Fig. 2 nicely confirmed such a view (Gatti, 2012; Monza et al., 2011). The $\mathrm{SF} \%$ values from the $\mathrm{C}$ atoms other than those directly involved in the $\mathrm{C} 1-\mathrm{C} 6$ bond increase with decreasing doublebond character and bond-electron localization for such a bond, on passing from cyclohexene to 1,3-cyclohexadiene and then to benzene. As expected, the SF contribution from the next-neighbour atoms $\mathrm{C} 2$ and $\mathrm{C} 5, \mathrm{SF}_{\mathrm{nn}} \%$, is significantly larger than that of the other farthest atoms, $\mathrm{SF}_{\mathrm{ot}} \%$. Conversely, the contribution from the two bonded atoms, $\mathrm{SF}_{\mathrm{ba}} \%$, linked through the b.c.p. taken as $r p$, was found to decrease along the series, although in a less evident way than the corresponding $\mathrm{SF}_{\mathrm{nn}} \%$ and $\mathrm{SF}_{\mathrm{ot}} \%$ augmentations, because the $\mathrm{H}$ atoms linked to the 'bonded atoms' also play a non-negligible role. All such illustrated trends were also followed by the corresponding SF absolute values (not shown in the figure, but see Monza et al., 2011; Gatti, 2012). The SF and SF\% values also visibly reflect the electron-delocalization asymmetry induced by the presence and location of the second double bond in 1,3cycloexadiene (Fig. 2). The SF\% patterns shown in the first row of Fig. 2 reveal that $\pi$-delocalization effects are small in the molecular plane, but clearly detectable even when $\pi$ electrons play an indirect role. However, when the $r p$ is moved above or below the molecular plane, the $\pi$-electrons may enter directly into the play and the effects of electron delocalization turn out to be largely enhanced. In the second row of Fig. 2,

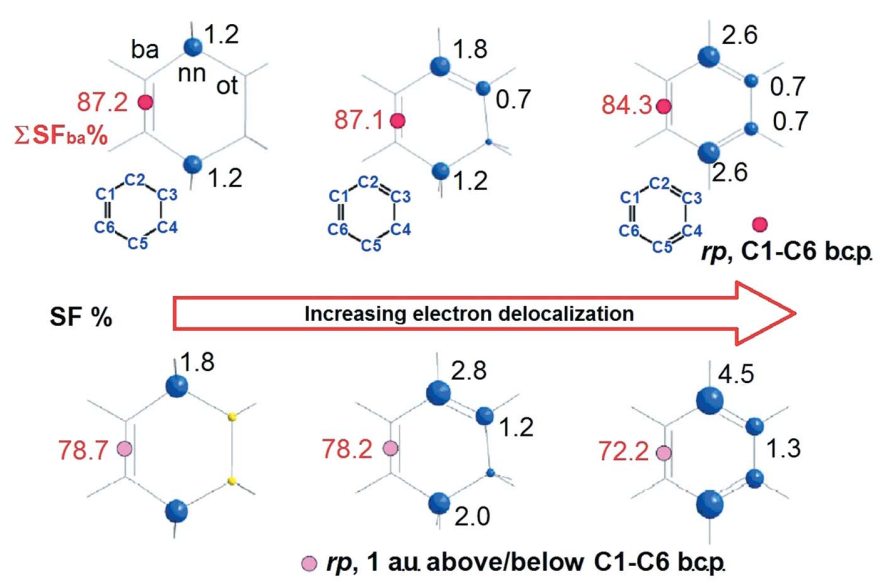

Figure 2

Source Function analysis of electron-delocalization effects in an unsaturated six-membered ring system with increasing $\pi$-electron conjugation. From left to right, SF\% data for cyclohexene, 1,3cyclohexadiene and benzene. The reference point is (top) at the b.c.p. of the shortest $\mathrm{CC}$ bond and (bottom) at points located \pm 1 a.u. above the molecular plane and displaced along the b.c.p. major axis direction. SF\% contributions for the next-neighbors, $n n$, and the farthest $\mathrm{C}$ atoms (denoted as 'others', ot) are given numerically and displayed as spheres whose volume is proportional to the contribution magnitude (blue, positive; yellow, negative). The sum of SF\% contributions from the two bonded atoms, $b a$, whose relevant b.c.p. is taken as the reference point, is also numerically reported (adapted from Fig. 1 and Scheme 1, from Monza et al., 2011 with permission; Copyright 2011, American Chemical Society). 
the $\mathrm{SF}_{\mathrm{nn}} \%$ and $\mathrm{SF}_{\mathrm{ot}} \%$ trends are shown when the $r p$ is moved 1 a.u. above/below the molecular plane and in the direction of the major axis of the $\pi$-electron distribution. Any $r p$ displacement along such a direction would lead to an increase in the electron-delocalization effects, but the 1 a.u. value was selected as a convenient $r p$ because the $\pi$-electron distribution has roughly a maximum at this distance from the plane.

Three more issues are worth being mentioned here. The first is that the capability of the SF to reflect $\pi$-electron delocalization is fully independent of the possible $\sigma / \pi$ separation of the ED. Actually, the SF analysis, in the reported example, has been applied to the total ED. Such a separation enables each total SF contribution to be partitioned in a term due to the $\sigma$ density and in a term due to the $\pi$-density (for a quantitative analysis see Gatti, 2012). However, the same values for the total SF and SF\% contributions would also be obtained by using an equivalent $\mathrm{ED}$, whose numerical values are given on a convenient grid, rather than in analytical form and in terms of molecular orbital contributions, and where $\sigma$ - and $\pi$-densities are no longer separable. This important property ensures application of the method to the ED derived from experiment and, irrespective of the ED origin, also to non-planar systems (Gatti et al., 2016) as in both these situations the $\sigma / \pi$ separation is not feasible. The second point to be recalled is that the SF patterns in $\pi$-delocalized systems were always found to essentially comply with the description of electron delocalization obtained from the delocalization indices (DIs). So, it was not a surprise to find a truly excellent correlation between a measure of local aromaticity based on DIs, the Fermi hole delocalization density (FHDD) index, and a novel formally analogous measure, based on the SF values and named SFLAI (Source Function Local Aromaticity Index; Monza et al., 2011). SFLAI, differently from FHDD, is clearly also applicable to experimentally derived ED distributions. Another facet to be emphasized is that the results of the SF analysis, including those specifically related to the electron-delocalization effects, appear to be generally rather stable against the use of different computational models and basis sets of different quality (Monza et al., 2011).

\subsection{Addressing criticisms about the SF ability to reveal electron-delocalization effects}

Following some interesting remarks from a referee, we now clarify and discuss a few controversial aspects that may raise doubts on the factual capability of the SF analysis to detect electron-delocalization effects. Generally speaking, one might object that the occurrence of delocalized sources does not necessarily imply electron-delocalization effects, as such types of sources are indeed the standard outcome when the electron density is reconstructed at the b.c.p.s associated with weak or moderately weak interactions, such as, for instance, hydrogen bonds (Gatti et al., 2003; for a general review, see Gatti, 2012). However, our present aim is to verify whether the otherwise almost localized sources of a covalently bonded system may still reflect minimal, yet appreciable changes in their numerical values, with known increasing/decreasing electron delocalization through its covalently bonded network. In other words, we were interested in differential SF values and patterns, rather than in their absolute counterparts. Therefore, we have restricted our analysis in $\$ 2.1$ to similarly covalently bonded systems, all characterized by well localized SF patterns and differing only in the number of delocalizable electrons (and of $\mathrm{H}$ atoms). One may then object that the trends we observed for the SF contributions from bonded and more distant atoms might not be related to the increased electron delocalization, but just to the significant geometry change caused by such an effect. While the indirect role of geometry change cannot be denied [see the expression of the local source in equation (2)], we have verified whether similar differential SF patterns could be obtained for the benzene, 1,3-cyclohexadiene and cyclohexene series also in terms of the electron density (evaluated at the DFT geometry) of the independent atomic model (IAM). ${ }^{1}$ The IAM density is the sum of undistorted atomic densities of the neutral atoms composing the system. Therefore, it should not contain any information about chemical bonding and, a fortiori, about interatomic electron correlation. Such a double lack of information might in principle produce any SF outcome, including a compensation of effects yielding individual SF patterns similar to those recovered from the DFT density. More unlikely, though still possible, one might even obtain a similar ordering of $\mathrm{SF}_{\mathrm{ba}} \%, \mathrm{SF}_{\mathrm{nn}} \%, \mathrm{SF}_{\mathrm{ot}} \%$ sources for the three systems, despite the lack of any electrondelocalization effect in the IAM density. On the other hand, any relevant discrepancy between the IAM and the DFT results would lend support to the ability of the SF to detect electron correlation effects. The interested reader can find a number of technical details and the full set of SF and SF\% values underlying the IAM study in the supporting information, while we discuss here only the main results. Being related to a 'non-bonding' electron density, the IAM b.c.p. density values are $\sim 30 \%$ lower than those found at the corresponding DFT b.c.p.s (shown in Fig. 2). The IAM SF absolute values would therefore be comparably lower if the SF\% values were identical for the two densities. This is not the case, especially for the $\mathrm{SF}_{\mathrm{nn}} \%$ and $\mathrm{SF}_{\mathrm{ot}} \%$ values, which are significantly higher, in some cases even by 2-3 times, for the non-bonded IAM model. The result is not surprising, as the lack of covalent bonding (or of any energetically important bonding interaction) is known to enhance the importance of the farthest regions in reconstructing the density at the associated b.c.p. Even more importantly, the trend of the $\mathrm{SF}_{\mathrm{nn}} \%$ and $\mathrm{SF}_{\mathrm{ot}} \%$ IAM values does not match that obtained with the DFT density for the cyclohexene/1,3-cyclohexadiene/benzene series, therefore being at variance with that anticipated by the increasing electron delocalization along the series. In particular, the $\mathrm{SF}_{\mathrm{nn}} \%$ and $\mathrm{SF}_{\mathrm{ot}} \%$ IAM values for cyclohexene, both at b.c.p. and at 1 a.u. above/below it, rather than being the smallest along the series, turn out to be significantly larger

\footnotetext{
${ }^{1}$ This is the so-called promolecule electron density, i.e. the IAM density evaluated at the 'true' molecular geometry, which is assumed here to be equal to that optimized at the DFT level.
} 
Table 1

Electron density and bond ellipticity values at $\mathrm{CC}$ b.c.p.s in benzene crystal for different multipolar model densities.

Data are reported for the multipolar model not including hexadecapole functions on $\mathrm{C}$ (No_Hex model) and, in parentheses and in italic, also for the model including hexadecapole functions on $\mathrm{C}$ (Hex_model). Standard deviations refer to the uncertainties estimated by the multipolar model in both cases.

\begin{tabular}{lll}
\hline Bond $\dagger$ & $\rho$ (b.c.p.) $\left(\mathrm{e} \AA^{-3}\right)$ & $\varepsilon$ \\
\hline $\mathrm{C} 1-\mathrm{C} 2$ & $2.26(2)[2.24(1)]$ & $0.23(0.02)$ \\
$\mathrm{C} 2-\mathrm{C} 3$ & $2.28(2)[2.28(1)]$ & $0.23(0.14)$ \\
$\mathrm{C} 1-\mathrm{C} 3^{\prime}$ & $2.21(2)[2.22(2)]$ & $0.23(0.16)$ \\
\hline
\end{tabular}

$\dagger$ Atom labels as in Fig. 3.

than those for 1,3-cyclohexadiene and benzene, which are instead very much alike. ${ }^{2}$ Such an outcome has two important consequences. On the one hand, it further validates the ability of the SF tool to reveal electron-delocalization effects, provided it is applied to a reasonable electron density, and on the other hand it rules out the geometric role as solely responsible for the changes in the SF patterns along the investigated series of compounds. A final important aspect still needs to be mentioned. Evaluation of the SF and SF\% atomic values implies the determination of atomic basin boundaries and the subsequent integration of the LS within the thus defined atomic basin. In the case of the promolecule density, we thought it reasonable to integrate the IAM LS within the atomic basin determined at the DFT level, so as not to mix two deficiencies: that due to the SF reconstruction of a fictitious density and that arising from the use of fictitious atomic boundaries, which implies incorrect partitioning of the reconstructed density into atomic contributions. Indeed, if the zero-flux QTAIM recipe is applied to the IAM density, atoms are obtained which are significantly away from neutrality (typically by $0.1-0.25 e^{-}$in our case), and therefore at variance with the nature of the non-interacting atoms inherent to the IAM density. ${ }^{3}$ This result clearly corroborates our choice of using the 'correct' DFT boundaries. Note that the SF\% values obtained by integrating the promolecular density in the atomic basins defined through this same density turn out, by chance, to follow the same qualitative trends found with the DFT density along the investigated series. Curiously enough, though not so infrequent in science, a qualitatively correct

\footnotetext{
${ }^{2}$ In the IAM model, the ED along the $\mathrm{C} 1-\mathrm{C} 6$ bond path (Fig. 2) has almost cylindrical symmetry, as denoted by the similar curvatures along the major and minor axes at the b.c.p. Moreover, the major axis, differently from what was anticipated by molecular orbital theory and from the DFT case, lies in the molecular plane. For the sake of comparison with the DFT density and probing the SF density reconstruction in what it is in reality, the plane associated with the $\pi$-electron distribution, we reconstructed the density at points located \pm 1 a.u. above the molecular plane and displaced, rather than along the b.c.p. major axis, along the b.c.p. minor axis, in the IAM case.

${ }^{3}$ One might argue that the more logical definition of an IAM atomic basin would be that corresponding to a fuzzy partitioning, one where the ED at each point is partitioned among atoms according to their own ED contribution at the point. For the sake of a reliable comparison, such a choice would however imply the adoption of a similar partition for the DFT density and therefore make use of an arbitrary rather than a rigorous quantum definition of atomic basin in apportioning the SF contributions.
}

Table 2

Benzene crystal: $\mathrm{SF} \%$ average values for the reconstruction of the electron density at the CC b.c.p.s and at reference points rps located above/below the molecular plane in the direction of the $\pi$-electron distribution of the CC bonds.

The reported values for $\mathrm{SF}_{\mathrm{ba}} \%, \mathrm{SF}_{\mathrm{nn}} \%$ and $\mathrm{SF}_{\mathrm{ot}} \%$ are averages over the $\mathrm{CC}$ bonds of the ring. Standard deviations relative to this average are given in parentheses. The symbols $b a, n n$ and ot denote, respectively, the contributions from the two mutually bonded atoms associated with the b.c.p., those from their nearest-neighbor $\mathrm{C}$ atoms and those from the other $\mathrm{C}$ atoms in the ring.

\begin{tabular}{lllll}
\hline$r p$ & $\rho$ type & $\mathrm{SF}_{\mathrm{ba}} \%$ & $\mathrm{SF}_{\mathrm{nn}} \%$ & $\mathrm{SF}_{\mathrm{ot}} \%$ \\
\hline b.c.p. & Exp (No_Hex model) & $86.2(2)$ & $5.2(1)$ & $1.5(0)$ \\
& Exp (Hex_model) & $86.2(2)$ & $5.0(1)$ & $1.3(0)$ \\
& Ab initio, periodic & $84.7(1)$ & $5.1(0)$ & $1.3(0)$ \\
\pm 1 a.u. $\neq$ & Ab initio, in vacuo $\dagger$ & 84.3 & 5.3 & 1.5 \\
& Exp (No_Hex model) & $73.5(3)$ & $9.5(2)$ & $3.1(1)$ \\
& Exp (Hex_model) & $72.6(8)$ & $9.3(6)$ & $2.7(2)$ \\
& Ab initio, periodic & $71.3(1)$ & $8.8(0)$ & $2.5(0)$ \\
& Ab initio, in vacuo $\dagger$ & 72.2 & 9.1 & 2.6 \\
\hline
\end{tabular}

† Data with in vacuo optimized geometry $\left(D_{6 h}\right.$ symmetry; Monza et al., 2011). $\ddagger$ For these out-of molecular plane $r p s, \mathrm{SF}_{\mathrm{ba}} \%, \mathrm{SF}_{\mathrm{nn}} \%$ and $\mathrm{SF}_{\mathrm{ot}} \%$ values are averaged both with respect to the $\mathrm{CC}$ bonds of the ring and to their location above ( +1 a.u.) and below $(-1$ a.u.) the plane. Distinct values for such locations are shown in Fig. 3 relative to only one bond $(\mathrm{C} 1-\mathrm{C} 2)$.

result may also be achieved through the lucky combination of several deficiencies in the adopted model.

2.3. Crystalline systems: $a b$ initio and experimentally derived electron densities of benzene and naphthalene molecular crystals

In order to explore whether the SF tool may also be applied to detect electron-delocalization effects in the condensed phase, using either ab initio or experimentally derived EDs, we considered first two prototypical $\pi$-conjugated systems, namely benzene and naphthalene molecular crystals. For such crystals very accurate charge-density quality X-ray diffraction data are available, while the corresponding periodic ab initio EDs were calculated in our study at the experimental geometries (cell parameters and atomic positions) and the DFT/B3LYP/6-311G** level. Benzene molecules crystallize in the centrosymmetric $P b c a$ space group, with half a molecule in the asymmetric unit and four molecules in the unit cell, whereas naphthalene molecules crystallize in the space group $P 2_{1} / c$, with half a molecule in the asymmetric unit and two molecules in the unit cell. The benzene ED was derived from single-crystal X-ray and neutron diffraction data by Bürgi et al. (2002) and that of naphthalene from the $135 \mathrm{~K} \mathrm{X}$-ray diffraction dataset measured by Oddershede \& Larsen (2004). Technical details on the derivation of benzene and napthalene EDs from the multipolar refinement of their X-ray diffraction datasets, on the evaluation of their ab initio periodic wavefunctions and on the calculation of SF contributions for both types of EDs, are reported in the supporting information. It is worth mentioning here that two different multipolar models have been considered in the case of benzene crystals, one including (Hex_model) and another not including (No_Hex model) hexadecapole functions on the $\mathrm{C}$ atoms. The first model gives better statistical agreement factors, but also 
predicts an ellipticity value which is too low for one of the three unique $\mathrm{CC}$ bonds in the six-membered ring, while the No_Hex model describes all these bonds similarly (see Table 1 ), as anticipated by the lack of strong non-covalent interactions between benzene molecules in the crystal (Bürgi et al., 2002). The bond ellipticity $\varepsilon$ [where $\varepsilon=\left(\lambda_{1} / \lambda_{2}-1\right)$ and $\lambda_{1}$ and $\lambda_{2}$ are the curvatures of the ED at the b.c.p. in a perpendicular direction to the bond path] measures the (extent of) departure from cylindrical symmetry of the electron distribution along the bond. The ED decreases more slowly in the direction of the $\pi$ distribution; the larger the double-bond character of a bond in a $\pi$-conjugated system, the lower the value of its $\lambda_{2}$ curvature and the larger the value of its ellipticity (Bader et al., 1983). As shown in Table 1, bond ellipticities, being curvatures of the ED, are very sensitive to the adopted multipolar model, while the ED values at the b.c.p. are much more stable against the model choice. SF\% data for benzene crystals, averaged over the ring $\mathrm{CC}$ bonds, are listed in Table 2 for the multipolar models and for the $a b$ initio ED, while Fig. 3 pictorially shows the $\mathrm{SF} \%$ data for the rps taken at one given $\mathrm{CC}$ b.c.p. $(\mathrm{C} 1-\mathrm{C} 2$ b.c.p.) and at 1 a.u. above and below the b.c.p. along the eigenvector associated with $\lambda_{2}$.

Data shown in Table 2 are particularly revealing. In spite of the fact that we are looking at subtle effects due to electron delocalization, agreement between SF\% values derived from $\mathrm{X}$-ray diffraction data and those obtained from $a b$ initio periodic wavefunctions is remarkable. Such a result applies to the various types of $\mathrm{SF} \%$ contributions $\left(\mathrm{SF}_{\mathrm{ba}} \%, \mathrm{SF}_{\mathrm{nn}} \%\right.$ and $\mathrm{SF}_{\mathrm{ot}} \%$ ) and regardless of whether the $r p$ lies on the molecular plane or above/below it. The No_Hex and the Hex models behave similarly for the ED reconstruction at the b.c.p., but they are found to (slightly) differ when the $r p$ is displaced out of the molecular plane. The Hex_model, in fact, exhibits standard deviations which are 2-3 times larger than the corresponding uncertainties in the No_Hex model. This is

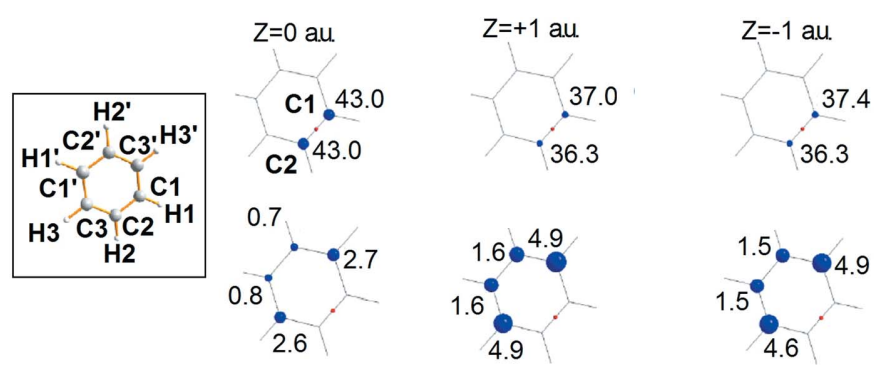

Figure 3

X-ray derived Source Function percentage patterns reflecting electrondelocalization effects in benzene crystal. The data refer to the No_Hex multipolar model electron density. The graphical inset on the leftmost part of the figure highlights the decrease in molecular symmetry upon packing and introduces the atomic labelling used in Table 1. Top row: Source Functions percentages (SF\%) from the $\mathrm{C} 1$ and $\mathrm{C} 2$ atoms reconstructing the electron density at a reference point located at their intervening $\mathrm{C} 1-\mathrm{C} 2$ b.c.p. $(z=0$ a.u.) and at reference points located \pm 1 a.u. $(z= \pm 1$ a.u.) above/below the molecular ring and displaced along the $\mathrm{C} 1-\mathrm{C} 2$ bond major axis. Bottom row: $\mathrm{SF} \%$ contributions from the nearest-neighbors and from the other $\mathrm{C}$ atoms at the same reference points of the first row. Reference point positions or their projections on the molecular ring are denoted by a red dot. likely due to the quite different and small bond ellipticity value predicted for one of the three unique $\mathrm{CC}$ bonds of benzene (Table 1). The large non-uniformity of the bond ellipticity values along the ring $\mathrm{CC}$ bonds found for the Hex_model denotes an increase in the $\pi$-distribution dissimilarity of these bonds, implying a larger distinction, hence a larger standard deviation, for the out-of-plane Hex_model $\mathrm{SF}_{\mathrm{ba}} \%, \mathrm{SF}_{\mathrm{nn}} \%$ and $\mathrm{SF}_{\mathrm{ot}} \%$ averages. The close resemblance of the in vacuo (geometry optimized, $D_{6 h}$ symmetry) and in crystal $a b$ initio $\mathrm{SF} \%$ values, and the small standard deviations of $a b$ initio crystalline SF\% data corroborate the lack of strong non-covalent interactions between benzene molecules in the crystal and agree with the quite small geometry perturbation induced by crystal packing. Inspection of Fig. 3 confirms the small geometric perturbation in the ring and the large similarity of the electron density, and hence of the electron distribution SF reconstruction, above and below the molecular plane for the benzene molecule in the crystal when analysed through the No_Hex model.

Experimentally derived and ab initio $\mathrm{SF} \%$ results for the naphthalene crystal are reported in Table 3, along with those for the naphthalene molecule in vacuo. Naphthalene may be seen as composed of two fused aromatic 6MRS (sixmembered rings), with the SF tool providing quite an interesting description of their mutual influence, whose full details and rationalization may be found in Monza et al. (2011). Here, we note that fusion of two benzenoid rings leads to a $D_{2 h}$ symmetry molecule and to four unique CC bonds, differing in number, from two up to four, and location of their nearest neighbor atoms. The latter may either belong to the same ring as the bond under examination or rather to the other ring, or be common to both of them. The four unique bonds differ as for their $\mathrm{CC}$ bond distances, delocalization indices and SF contribution patterns. In particular, the $\mathrm{SF}_{\mathrm{nn}} \%$ value increases with increasing number of nearest-neighbor $\mathrm{C}$ atoms, each of them bringing a contribution of $\sim 2.5 \%$ to the ED value at the b.c.p. The central $\mathrm{C} 3-\mathrm{C}^{\prime}$ bond, with the four nearestneighboring $\mathrm{C}$ atoms, therefore has the highest $\mathrm{SF}_{\mathrm{nn}} \%$ value, $9.8 \%$, which is almost twice as large as the unique $\mathrm{CC}$ in benzene or the $\mathrm{C} 1-\mathrm{C} 2$ or $\mathrm{C} 1-\mathrm{C}^{\prime}$ bonds in naphthalene, while $\mathrm{C} 2-\mathrm{C} 3$ which has three nearest-neighbors exhibits an intermediate $\mathrm{SF}_{\mathrm{nn}} \%$ value of $7.3 \% . \mathrm{SF}_{\mathrm{ot}} \%$ values also increase relative to benzene, owing to the increased number of the other atoms, from two to eight in naphthalene, but the increment in the $\mathrm{SF}_{\text {ot }} \%$ value turns out to be clearly larger the less peripheral the bond whose $r p \mathrm{ED}$ is reconstructed.

The general increase of $\mathrm{SF}_{\mathrm{nn}} \%$ and $\mathrm{SF}_{\mathrm{ot}} \%$ values implies a general decrease in $\mathrm{SF}_{\mathrm{ba}}$ \% contribution for all four unique bonds in naphthalene, except the $\mathrm{C} 1-\mathrm{C} 2$ bond that has a larger double bond character and is shorter than the $\mathrm{CC}$ bond in benzene. Its $\mathrm{SF}_{\mathrm{ba}}$ \% value of 85.3 is larger than that of benzene $(84.3 \%)$ and the largest among the four unique bonds in naphthalene. Similar trends, relative to the corresponding ones in benzene, are found when the $r p$ is moved above/below the molecular plane for the various $\mathrm{CC}$ bonds in naphthalene. By placing this molecule in the crystal, the molecular symmetry reduces to $C_{2 h}$ (Table 3 ). However, the bond 
Table 3

Naphthalene crystal and molecule: SF\% average values for the reconstruction of the electron density at the various CC b.c.p.s and at reference points $r p s$ located above/below the molecular plane in the direction of the $\pi$-electron distribution of the CC bonds.

$\mathrm{SF} \%$ and bond lengths $d$ : for each bond or couple of bonds the first row refers to values from experimentally derived multipolar ED, the second to data from periodic computations at crystal experimental geometry and the third one (in italics) to values from the in vacuo calculations (Monza et al., 2011) at $D_{2 h}$ symmetry optimized geometry. The in-crystal data for $\mathrm{SF}_{\mathrm{ba}} \%, \mathrm{SF}_{\mathrm{nn}} \%$ and $\mathrm{SF}_{\mathrm{ot}} \%$ are averages over the CC bonds, which are related by symmetry in the in vacuo $D_{2 h}$ molecule. Values in parentheses represent the standard deviations of these averages. For the out of the molecular plane rps, averages also include SF\% data for $r p s$ above ( +1 a.u.) and below ( -1 a.u.) such a plane. The symbols $b a, n n$ and $o t$ retain the same meaning as in Table 2.

\begin{tabular}{|c|c|c|c|c|c|c|c|c|}
\hline \multirow[b]{2}{*}{ Atom labels $\dagger$} & \multirow[b]{2}{*}{ Bond(s) } & \multirow[b]{2}{*}{$d, \AA$} & \multicolumn{3}{|l|}{ b.c.p. } & \multicolumn{3}{|l|}{ \pm 1 a.u. } \\
\hline & & & $\mathrm{SF}_{\mathrm{ba}} \%$ & $\mathrm{SF}_{\mathrm{nn}} \%$ & $\mathrm{SF}_{\mathrm{ot}} \%$ & $\mathrm{SF}_{\mathrm{ba}} \%$ & $\mathrm{SF}_{\mathrm{nn}} \%$ & $\mathrm{SF}_{\mathrm{ot}} \%$ \\
\hline \multirow{9}{*}{ 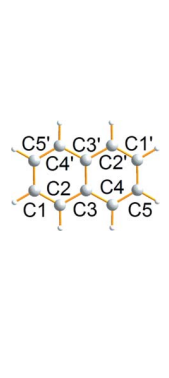 } & $\mathrm{C} 1-\mathrm{C} 2 / \mathrm{C} 4-\mathrm{C} 5 \ddagger$ & 1.374 & $86.3(0)$ & $4.2(1)$ & $2.4(1)$ & $73.6(1)$ & $7.3(1)$ & $4.8(1)$ \\
\hline & & 1.380 & 85.3 & 4.4 & 2.3 & 74.2 & 7.2 & 4.3 \\
\hline & \multirow{3}{*}{$\mathrm{C} 1-\mathrm{C}^{\prime}$} & 1.416 & 84.3 & 5.8 & 1.9 & $69.6(1)$ & $10.5(1)$ & $3.9(1)$ \\
\hline & & 1.416 & 83.6 & 5.8 & 1.7 & $69.4(1)$ & $10.1(0)$ & $3.4(0)$ \\
\hline & & 1.424 & 83.7 & 5.9 & 1.9 & 70.4 & 10.1 & 3.7 \\
\hline & $\mathrm{C} 2-\mathrm{C} 3 / \mathrm{C} 3-\mathrm{C} 4 \ddagger$ & 1.427 & 82.5 & 7.3 & 3.0 & 68.3 & 12.2 & 5.9 \\
\hline & \multirow[t]{3}{*}{$\mathrm{C} 3-\mathrm{C}^{\prime}$} & 1.422 & 81.6 & 9.8 & 3.3 & $64.4(0)$ & $17.3(0)$ & $6.5(0)$ \\
\hline & & 1.422 & 81.2 & 9.5 & 2.9 & $64.5(0)$ & $16.5(0)$ & $5.6(0)$ \\
\hline & & 1.434 & 81.2 & 9.8 & 3.2 & 65.4 & 16.6 & 6.0 \\
\hline Benzene § & $\mathrm{C}-\mathrm{C}$ & 1.402 & 84.3 & 5.3 & 1.5 & 72.2 & 9.1 & 2.6 \\
\hline
\end{tabular}

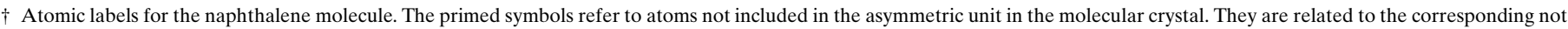

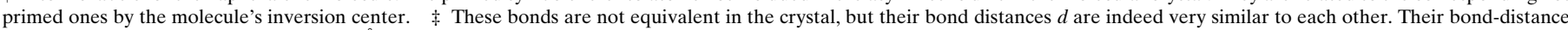
differences amount to 0.0002 and $0.0006 \AA$ for $\mathrm{C} 4-\mathrm{C} 5 / \mathrm{C} 1-\mathrm{C} 2$ and $\mathrm{C} 2-\mathrm{C} 3 / \mathrm{C} 3-\mathrm{C} 4$, respectively. $\S$ Data for benzene refer to the benzene molecule in vacuo, at $D_{6 h}$ optimized geometry (Monza et al., 2011).

distances of the bonds which have become inequivalent in the crystal hardly differ, their differences amounting to 0.0002 and $0.0006 \AA$ for $\mathrm{C} 4-\mathrm{C} 5 / \mathrm{C} 1-\mathrm{C} 2$ and $\mathrm{C} 2-\mathrm{C} 3 / \mathrm{C} 3-\mathrm{C} 4$, respectively. Inspection of the data in Table 3 reveals that the naphthalene molecule in the crystal displays the same ordering of SF\% contributions as for the molecule in vacuo. This is indeed a remarkable result as such an agreement applies both to the data derived from X-ray diffraction and to those from the $a b$ initio wavefunction, and to all types of contributions, $\mathrm{SF}_{\mathrm{ba}} \%, \mathrm{SF}_{\mathrm{nn}} \%$ and $\mathrm{SF}_{\mathrm{ot}} \%$, regardless of whether the rps are taken at the b.c.p.s or displaced above/ below them. Not only is the same ordering preserved, but also an almost quantitative agreement is recovered. Such a result complies with the weak intermolecular interactions present in the crystal and the scarce effect they have on molecular geometry and on the electron delocalization mechanisms. Besides, it also shows that the ED derived from experiment accurately reproduces the subtle effects induced by electron delocalization on the SF\% contributions - effects that in the case of naphthalene result in a bond differentiation, and in a characteristic discrimination and ordering of the corresponding SF\% patterns. In the next subsection we explore whether such promising results also apply to a more challenging and less paradigmatic case.

2.4. Crystalline systems: $a b$ initio and experimentally derived electron densities of $( \pm)$-8' -benzhydrylideneamino-1, $\mathbf{1}^{\prime}$ binaphthyl-2-ol (BAB) crystal

The $( \pm)$-8'-benzhydrylideneamino-1,1'-binaphthyl-2-ol molecule (hereinafter BAB, Scheme 1), whose crystal structure was reported by Vyskočil et al. (2002), crystallizes in the
$P 2_{1} / c$ group, with four molecules per cell and 58 atoms (236 electrons) in the asymmetric unit. Since this racemic hydroxyimine molecule forms excellent quality crystals, Farrugia et al. (2009) were able to study their charge density experimentally, using Mo $K \alpha$ X-ray diffraction at $100 \mathrm{~K}$, in order to investigate the electronic features of several interesting and weak peri-C $\cdots \mathrm{N}, \mathrm{CH} \cdots \pi, \mathrm{H} \cdots \mathrm{H}$ and $\mathrm{C}(\pi) \cdots \mathrm{C}(\pi)$ intramolecular interactions, suggested on geometrical grounds by the previous structural investigation (Vyskočil et al., 2002).

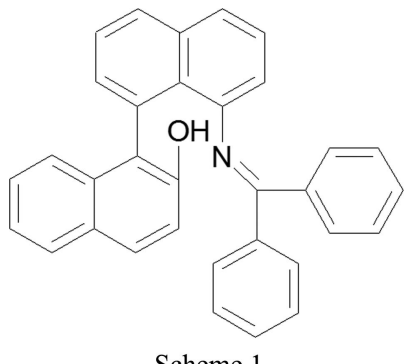

Based on the structure factors obtained by Farrugia et al. (2009) we have re-examined the experimental charge density of BAB and supplemented their DFT calculations for the molecule in vacuo with DFT periodic calculations in the bulk (full details on the adopted multipolar model strategy and on the performed quantum-mechanical calculations are reported in the supporting information). Our interest in BAB is motivated here by the presence of two benzyl and two naphthyl moieties in the same molecule, but substituted differently. Therefore, BAB represents an excellent case for investigating whether and to what extent the SF patterns found for benzene and naphthalene are transferable to the benzyl and naphthyl 
Table 4

$\mathrm{BAB}$ crystal: experimentally derived $\mathrm{SF} \%$ average values for the reconstruction of the electron density at the various $\mathrm{CC}$ b.c.p.s of the two benzyl rings of the molecular unit and at reference points located above/below the molecular planes of the two rings, in the direction of the $\pi$-electron distribution of the $\mathrm{CC}$ bonds.

Benzene crystal data are also reported for the sake of comparison. Data for ring $A$ and $B$ are evaluated as averages over the six $\mathrm{CC}$ bonds of each ring either including or excluding from the average the $\mathrm{CC}$ bonds having one of the two $\mathrm{C}$ atoms linked to the bridging $\mathrm{C}$ atom. Values in parentheses represent the standard deviations of these averages. For the out-of-the-molecular plane rps, averages also include SF\% data for $r p s$ above ( +1 a.u.) and below ( -1 a.u.) such a plane. The symbols $b a, n n$ and $o t$ retain the same meaning as in Table 2

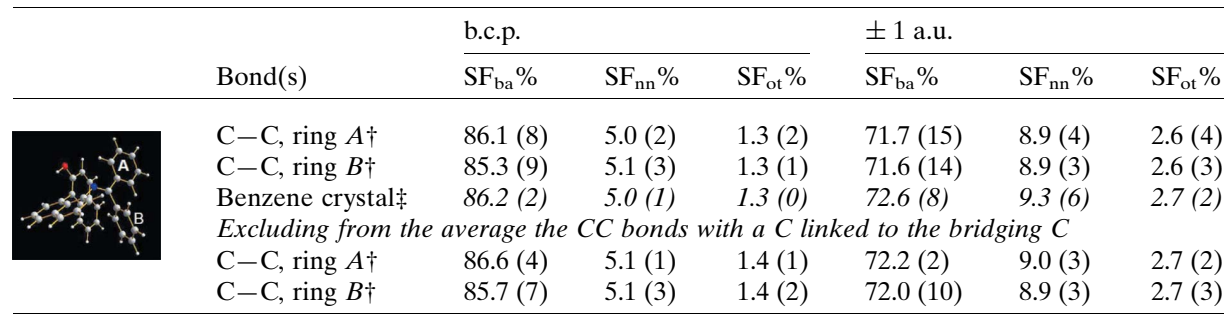

$\dagger$ Ring labels shown in the scheme of this table. $\$$ Data for the benzene crystal refer to the experimentally derived electron density and to the Hex_model, for the sake of comparison with the BAB adopted multipolar model which also includes hexadecapole functions on atoms other than $\mathrm{H}$

Table 5

$\mathrm{BAB}$ crystal: experimentally derived SF\% average values for the reconstruction of the electron density at the various $\mathrm{CC}$ b.c.p.s of the least perturbed rings (rings I) of the naphthyl and naphthylol moieties and at reference points located above/below the molecular planes of these two rings, in the direction of the $\pi$-electron distribution of the $\mathrm{CC}$ bonds.

Naphthalene crystal, experimentally derived data, are also reported for comparison (NAR columns).

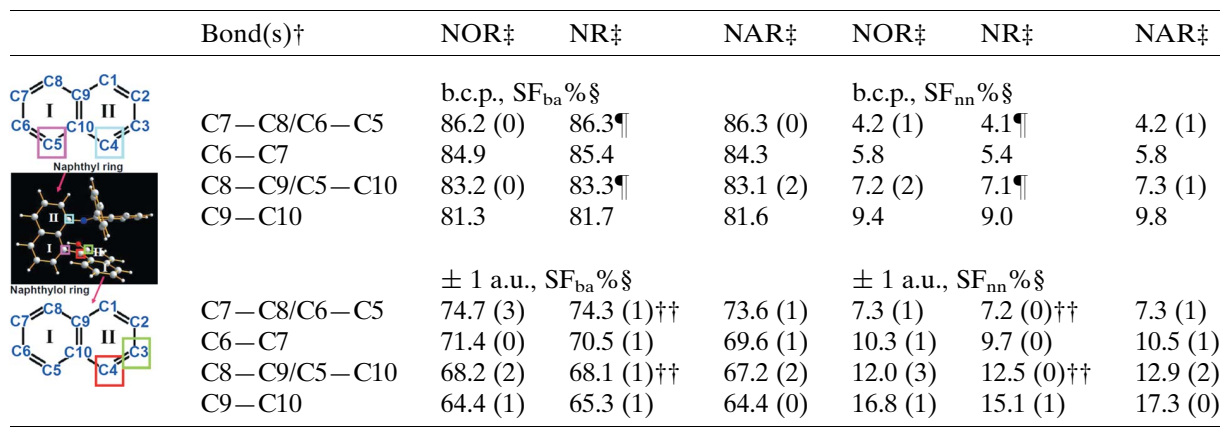

$\dagger$ Bonds and rings are labelled according to the scheme displayed in the table. Adopting common atomic labelling for the naphthalene, naphthyl and naphthylol moieties enables easier comparison between the SF\% values of the corresponding bonds. The $\mathrm{C}$ atoms whose linked $\mathrm{H}$ atoms have been substituted by $\mathrm{C}, \mathrm{N}$ or $\mathrm{O}$ in the naphthyl or naphthylol moieties of $\mathrm{BAB}$ are enrolled in a square. Both rings of the naphthyl moiety exhibit one $\mathrm{H}$ replacement relative to naphthalene, but ring I is considered as the least perturbed because the electronegativity of the replacing atom, $\mathrm{C}$, is more similar to that of $\mathrm{H}$, than it is that of $\mathrm{N} . \quad \ddagger \mathrm{NOR}=$ naphthylol ring $\mathrm{I} ; \mathrm{NR}=$ naphthyl ring $\mathrm{I} ; \mathrm{NAR}=$ naphthalene ring. $\S \mathrm{SF} \%$ data are averages of those of the bonds listed in column 2. Values in parentheses represent the root mean square deviation from the average. For the out-of-the-molecular plane $r p s$, averages also include SF\% data for $r p s$ above ( +1 a.u.) and below ( -1 a.u.) such a plane The symbols $b a$ and $n n$ retain the same meaning as in Table 2. Data refer only to the bond not including the substituted C5. SF\% data for the $\mathrm{C} 6-\mathrm{C} 5$ bond are 84.5 and 4.2 for $\mathrm{SF}_{\mathrm{ba}} \%$ and $\mathrm{SF}_{\mathrm{nn}} \%$, respectively, and those for the $\mathrm{C} 5-\mathrm{C} 10$ bond are 81.5 and 6.7 for $\mathrm{SF}_{\mathrm{ba}} \%$ and $\mathrm{SF}_{\mathrm{nn}} \%$, respectively. $\dagger \dagger$ Data refer only to the bond not including the substituted $\mathrm{C} 5 . \mathrm{SF} \%$ ( \pm 1 a.u.) averaged data for the $\mathrm{C} 6-\mathrm{C} 5$ bond are 71.6 and 7.2 for $\mathrm{SF}_{\mathrm{ba}} \%$ and $\mathrm{SF}_{\mathrm{nn}} \%$, respectively, and those for the $\mathrm{C} 5-$ $\mathrm{C} 10$ bond are 65.9 and 11.3 for $\mathrm{SF}_{\mathrm{ba}} \%$ and $\mathrm{SF}_{\mathrm{nn}} \%$, respectively.

moieties of BAB, and also whether chemical substitution has an easily detectable impact on these patterns. Furthermore, it enables the assessment of the internal consistency of the experimental data and the adopted multipolar model through comparison of the SF patterns of its two benzyl and two naphthyl groups. Such analysis and comparisons attract further interest and significance as they involve ED distributions derived from experiments carried out under rather different, although always appropriate, operating conditions (temperature, beam intensity, crystal size, data collection strategy, data quality, multipolar model etc.).

Table 4 reports the average SF experimental patterns for the $\mathrm{CC}$ bonds in the two benzyl rings of the $\mathrm{BAB}$ crystal and compares them with those in the benzene crystal. Data for either of the two benzyl rings of BAB are evaluated either including or not including in the average the $\mathrm{CC}$ bonds with one of the two $\mathrm{C}$ atoms of the bond linked to the bridging $\mathrm{C}$ atom. Analogously to the benzene crystal, $\mathrm{SF} \%$ values for $r p s$ displaced from the plane of the ring refer to averages including data for rps locations above and below the ring. Results shown in Table 4 clearly indicate that the two benzyl rings of $\mathrm{BAB}$ exhibit electrondelocalization SF patterns almost equal to those in the benzene crystal, regardless of the chosen $r p$ location. As a consequence, the data for the two rings also show an excellent degree of internal consistency.

The effect of substitution is revealed by the reported deviations from the SF\% averages. For both $\mathrm{BAB}$ benzyl rings these deviations are 1-4 times larger than for benzene and, as absolute values, higher for the $\mathrm{SF}_{\mathrm{ba}} \%$ components. Nonetheless, they are limited and turn out to be largely reduced (Table 4) when the $\mathrm{SF} \%$ data for $\mathrm{CC}$ bonds having a $\mathrm{C}$ linked to the bridging $\mathrm{C}$ atoms are not included in the average.

The electron-delocalization features in the naphthyl and naphthylol 10-membered rings (10MRs) are compared with those of naphthalene in Tables 5 and 6 , using a common set of atomic labels to symbolize the $10 \mathrm{MRs} \mathrm{C}$ atoms in the three systems. It is informative to split such a comparison into two steps. First we focus on the supposedly 'least-perturbed' 6MRS of the two 10MRs, relative to naphthalene. They are both denoted as ring I (leftmost column, Table 5) and are either characterized by no substitution (naphthylol ring) or by just one substituted $\mathrm{H}$ (naphthyl ring) relative to the corresponding 6MRS in naphthalene. For the naphthyl ring, where each $6 \mathrm{MR}$ exhibits a substituted $\mathrm{H}$ atom, ring $\mathrm{I}$ is assumed to be the least perturbed because the electronegativity of the 
Table 6

$\mathrm{BAB}$ crystal: experimentally derived $\triangle \mathrm{SF} \%$ values for the reconstruction of the electron density at the various CC b.c.p.s of the most perturbed rings (rings II) of the naphthyl and naphthylol moieties and at reference points located above/below the molecular planes of these two rings, in the direction of the $\pi$-electron distribution of the CC bonds.

$\Delta \mathrm{SF}_{x x} \%(x x=$ ba or $\mathrm{nn})$ values for each bond of rings II of BAB are evaluated relative to the Source Function contributions of the corresponding bond in naphthalene crystal, $\Delta \mathrm{SF}_{x x} \%=\mathrm{SF}_{x x} \%(\mathrm{BAB})-\mathrm{SF}_{x x} \%$ (naphthalene crystal, experimentally derived data, Table 3 ). The symbols $b a$ and $n n$ retain the same meaning as in Table 2 .

\begin{tabular}{|c|c|c|c|c|}
\hline Bond(s) $\dagger$ & \multicolumn{2}{|c|}{ NOR $\ddagger N R \ddagger$} & \multicolumn{2}{|c|}{ NOR $\ddagger \mathrm{NR} \ddagger$} \\
\hline & \multicolumn{2}{|c|}{ b.c.p., $\Delta \mathrm{SF}_{\mathrm{ba}} \% \neq$} & \multicolumn{2}{|c|}{ b.c.p., $\Delta \mathrm{SF}_{\mathrm{nn}} \% \ddagger$} \\
\hline $\mathrm{C} 1-\mathrm{C} 2$ & -0.1 & +0.9 & -0.3 & 0.0 \\
\hline $\mathrm{C3}-\mathrm{C} 4$ & -3.3 & -2.0 & -0.3 & 0.0 \\
\hline $\mathrm{C} 2-\mathrm{C3}$ & -2.1 & +0.2 & -0.5 & -0.4 \\
\hline $\mathrm{C} 1-\mathrm{C} 9$ & -0.1 & -0.1 & -0.1 & -0.1 \\
\hline C4-C10 & -1.8 & -1.9 & -0.6 & -0.7 \\
\hline \multirow[t]{2}{*}{$\mathrm{C} 9-\mathrm{C} 10$} & -0.3 & +0.1 & -0.4 & -1.0 \\
\hline & \multicolumn{2}{|c|}{ \pm 1 a.u., $\Delta \mathrm{SF}_{\mathrm{ba}} \% \neq$} & \multicolumn{2}{|c|}{ \pm 1 a.u., $\Delta \mathrm{SF}_{\mathrm{nn}} \% \ddagger$} \\
\hline $\mathrm{C} 1-\mathrm{C} 2$ & +0.6 & +0.9 & -0.8 & 0.0 \\
\hline C3-C4 & -4.5 & -2.4 & -0.7 & -0.2 \\
\hline $\mathrm{C} 2-\mathrm{C} 3$ & -2.1 & +1.3 & -0.8 & -0.8 \\
\hline $\mathrm{C} 1-\mathrm{C} 9$ & +0.9 & +0.8 & -0.2 & -0.2 \\
\hline C4-C10 & -2.3 & -2.8 & -1.4 & -1.6 \\
\hline $\mathrm{C} 9-\mathrm{C} 10$ & 0.0 & 0.9 & -0.6 & -2.1 \\
\hline
\end{tabular}

$\dagger$ Bonds and rings are labelled according to the drawing displayed in the leftmost column of Table 5. $\ddagger \Delta \mathrm{SF}_{x x}(x x=$ ba or $\mathrm{nn})$ data for each bond evaluated relative to the above/ below rps average values (both in $\mathrm{BAB}$ and in naphthalene crystal). NOR = naphthylol ring II; NR = naphthyl ring II; NAR = naphthalene ring. Bond labels and $\Delta \mathrm{SF}_{x x} \%(x x=$ ba or $\mathrm{nn}$ ) reported in bold are relative to bonds with one or two H-substituted carbon atoms in NOR and NR (for the $\mathrm{C} 2-\mathrm{C} 3$ bond, the bold mark refers only to NOR).

replacing atom, $\mathrm{C}$, is closer to that of the $\mathrm{H}$ than that of $\mathrm{N}$, the atom replacing $\mathrm{H}$ in ring II. Data shown in Table 5 suggest a remarkable similarity among the SF\% patterns of the three compared systems for all four types of bonds typical of the naphthalene moiety. Not only are the $\mathrm{SF}_{x x} \%(x x=$ ba or $\mathrm{nn})$ values similarly ordered, as a function of $\mathrm{CC}$ bond type, but they also exhibit an almost quantitative agreement in the three systems, which applies to $r p$ locations taken at the b.c.p.s and almost equally as well to those \pm 1 a.u. above/below the b.c.p.s.

The transferability of the typical electron-delocalization SF\% features of naphthalene (Monza et al., 2011) appears to be well proven for the least perturbed rings of the naphthalene-like moieties of BAB, but the effect of substitution at C5 in the naphthyl ring $\mathrm{I}$ is also easily spotted through careful analysis of these same features. Indeed, in the case of the naphthyl ring the values for $\mathrm{CC}$ bonds involving $\mathrm{C} 5$ (C6- C5 and $\mathrm{C} 5-\mathrm{C} 10)$ were purposely not included in the averages and the reported values refer only to their symmetry-related bonds in naphthalene $(\mathrm{C} 7-\mathrm{C} 8$ and $\mathrm{C} 9-\mathrm{C} 10)$. Data for bonds involving $\mathrm{C} 5$, listed in two footnotes of Table 5 for $r p=$ b.c.p. and for rps above/below b.c.p.s, respectively, clearly do not follow the trends, in particular the $\mathrm{SF}_{\mathrm{ba}} \%$ values. This result is not surprising because the $\mathrm{C} 5$ atom is directly involved in the determination of the $\mathrm{SF}_{\mathrm{ba}} \%$ value, which is different to the $\mathrm{SF}_{\mathrm{nn}} \%$ value where the involvement of $\mathrm{C} 5$ is indirect. $\mathrm{SF}_{\mathrm{ba}} \%$ values for bonds including $\mathrm{C} 5$ are lowered with respect to their symmetry-related bonds in naphthalene because of a lower $\mathrm{SF} \%$ contribution from the $\mathrm{C} 5$ atom, due to the replacement of its linked $\mathrm{H}$ atom by the more electronegative $\mathrm{C} 4$ atom of the naphthylol ring.

Table 6 lists SF\% pattern data for the 'most perturbed' rings of the naphthyl and naphthylol moieties of BAB. These rings are named rings II and their associated atomic labelling is shown in the leftmost column of Table 5. To better highlight the departures of $\mathrm{SF} \%$ values from those of naphthalene, data for each type of $\mathrm{CC}$ bond are reported as $\Delta \mathrm{SF}_{x x} \%(x x=$ ba or $\mathrm{nn})$ differences relative to the corresponding bond in naphthalene, where $\Delta \mathrm{SF}_{x x} \%=$ $\mathrm{SF}_{x x} \%$ (in $\mathrm{BAB}$ ) - $\mathrm{SF}_{x x} \%$ (in the naphthalene crystal, experimentally derived data, Table 3). Inspection of Table 6 clearly reveals that, even for the most perturbed rings, $\mathrm{SF} \%$ data for $\mathrm{CC}$ bonds whose linked $\mathrm{H}$ atoms have not been replaced closely match those for the corresponding bonds in naphthalene. However, larger and significant deviations of $\mathrm{SF} \%$ values are observed for those bonds involving one or two H-substituted $\mathrm{C}$ atoms. For the sake of clarity these bonds and related SF\% values are denoted in bold in Table 6. The largest departures, with values even exceeding $4 \%$, involve $\mathrm{SF}_{\mathrm{ba}} \%$ data and, in particular, those bonds where both $\mathrm{C}$ atoms have undergone replacement of their linked $\mathrm{H}$ atoms (compare $\mathrm{C} 3-\mathrm{C} 4$ versus $\mathrm{C} 2-\mathrm{C} 3$ and $\mathrm{C} 4-\mathrm{C} 10 \mathrm{SF} \%$ data in the naphthylol ring II). Indeed, since $\mathrm{H}$-atom substitution by higher electronegativity elements generally leads to a decrease in the $\mathrm{SF} \%$ contribution to the $\mathrm{SF}_{\mathrm{ba}} \%$ value by their associated $\mathrm{C}$ atoms, $\mathrm{H}$-substitution of both the $\mathrm{C}$ atoms involved in a bond leads to the reinforcement of such a $\mathrm{SF}_{\mathrm{ba}} \%$ decrease. H-substitution by more electronegative elements decreases rather than increases the $\mathrm{SF}_{\mathrm{ba}} \%$ values, because fewer electrons are available to the $\mathrm{C}$ atoms for bonding and the $\mathrm{CC}$ bond becomes much less covalent in nature. For homopolar bonds, a decreased bond covalency is mirrored by a decreased SF\% contribution from the bonded atoms to their intervening b.c.p. (Gatti, 2012) and leads, in general, to an increase in bond distances, which is precisely what we observe in the naphthyl and naphthylol rings when $\mathrm{CC}$ distances originally related by symmetry in naphthalene are compared. Upon $\mathrm{H}$-substitution, the $\mathrm{CC}$ b.c.p. location also becomes less symmetrical, a further indication of the slight departure from covalency. Clearly, the decrease of the $\mathrm{SF}_{\mathrm{ba}} \%$ values, induced by $\mathrm{H}$-substitution, should not be considered in this case as a sign of an electron-delocalization enhancement. The previously discussed decreasing $\mathrm{SF}_{\mathrm{ba}} \%$ values with increasing electron delocalization, as in the case of the cyclohexene, 1,3-cyclohexadiene and benzene series, may only apply when other (highly) disruptive factors are absent. Yet, the perturbation induced by $\mathrm{H}$-substitution with more electronegative elements is mostly local in character as it soon fades away through the electron delocalized network of bonds $\left(\Delta \mathrm{SF}_{\mathrm{nn}} \%\right.$ values are significantly smaller than $\Delta \mathrm{SF}_{\mathrm{ba}} \%$ values, Table 6 , and $\Delta \mathrm{SF}_{\mathrm{ot}} \%$ values, not reported in this Table, are negligible). 


\section{Conclusions}

From the evidence reported in this paper it is shown that, by making use of X-ray derived experimental electron densities, the Source Function descriptor is capable of detecting subtle effects due to $\pi$-electron delocalization in organic molecular crystals. Such effects are retrieved in amazingly quantitative agreement with those obtained through $a b$ initio periodic computations of the corresponding electron densities. When applicable, the electron delocalization effects also seem to be largely transferable from system to system, regardless of whether their underlying electron density distributions had been obtained from different crystal data sets and under different experimental conditions. This testifies to both the quality and consistency of such electron densities and the robustness of the SF features associated with electron-delocalization effects. As further proof it is indeed shown that despite the dominance of the two directly bonded atoms in reconstructing the electron density at their intervening b.c.p., the overall SF patterns implied by electron delocalization features in a given $\pi$ conjugated chemical framework are so distinctive that the effect of chemical substitution may be easily identified and quantified.

Although the capability of the SF to detect electron-delocalization effects can only be proved in a heuristic manner, this paper shows convincingly that using a promolecular rather than a proper quantum mechanical density leads to quite different trends in the SF\% patterns of covalently bonded, closely related systems, differing by their electron-delocalization possibilities (namely, the cyclohexene, 1,3-cyclohexadiene and benzene series). The promolecular density, at variance with the proper quantum density, appears to be unable to reproduce the SF trends anticipated by an increase in electron delocalization along this series, therefore challenging the hypothesis that the geometrical effect is playing the only role in the game. Use of an ED able to describe chemical bonding and electron-delocalization effects looks to be mandatory to recover those SF features that we have supposedly associated with electron delocalization, although one cannot exclude that, by error compensation, in some cases one might still obtain similar qualitative features through a promolecular density.

The Source Function, having the attractive feature of being applicable on a common and rigorous basis to electron densities derived either from experiment or theory, appears to have wider applications than those already explored for discussing the nature of a chemical bond in more or less conventional situations. Detection of electron-delocalization effects, as highlighted in this paper, is one such new direction, another being the recent extension of the Source Function machinery to retrieve the atomic sources of the electron spin density (Gatti et al., 2015, 2016). In both cases, the already feasible or potential (in the case of electron spin densities) applications to observations derived from X-ray or polarized neutron diffraction experiments look particularly appealing and promising.

\section{Related literature}

References cited in the supporting information include: Gatti (2010), Dovesi et al. (2009), Godbout et al. (1992), Volkov et al. (2006).

\section{Acknowledgements}

We wish to warmly thank Professor Sine Larsen and Professor Mark Spackman for providing us with the $135 \mathrm{~K} \mathrm{X}$-ray diffraction dataset of naphthalene crystal and the $110 \mathrm{~K} \mathrm{X}$-ray diffraction data set of benzene, respectively. We also thank the Danish National Research Foundation for partial funding of this work through the Center for Materials Crystallography (DNRF93). GS acknowledges financial support from the grant of the Government of the Russian Federation (No. 14.A12.31.0003). Computing time provided by the Italian supercomputing centre CINECA through LISA and ISCRAC grants was also very much appreciated.

\section{References}

Arfken, G. (1985). Mathematical Methods for Physicists. Academic Press: Orlando, Florida.

Bader, R. F. W. (1990). Atoms in Molecules: A Quantum Theory. Oxford University Press.

Bader, R. F. W. \& Gatti, C. (1998). Chem. Phys. Lett. 287, 233-238.

Bader, R. F. W., Slee, S., Cremer, D. \& Kraka, E. (1983). J. Am. Chem. Soc. 105, 5061-5068.

Bultinck, P. (2007). Faraday Discuss. 135, 347-365.

Bultinck, P., Rafat, M., Ponec, R., Van Gheluwe, B., Carbó-Dorca, R. \& Popelier, P. (2006). J. Phys. Chem. A, 110, 7642-7648.

Bürgi, H.-B., Capelli, S. C., Goeta, A. E., Howard, J. A. K., Spackman, M. A. \& Yufit, D. S. (2002). Chem. Eur. J. 8, 3512-3521.

Cocq, K., Lepetit, C., Maraval, V. \& Chauvin, R. (2015). Chem. Soc. Rev. 44, 6535-6559.

Cremer, D., Kraka, E., Slee, S., Bader, R. F. W., Lau, C. D. H., Nguyen Dang, T. T. \& MacDougall, P. J. (1983). J. Am. Chem. Soc. 105, 5069-5075.

Dovesi, R., Saunders, V. R., Roetti, C., Orlando, R., Zicovich-Wilson, C. M., Pascale, F., Civalleri, B., Doll, K., Harrison, N. M., Bush, I. J., D'Arco, P. \& Llunell, M. (2009). CRYSTAL09. University of Torino, Italy.

Engels, B., Schmidt, T. C., Gatti, C., Schirmeister, T., Reinhold, F. \& Fink, R. F. (2012). Struct. Bond. 147, 47-98.

Farrugia, L. J., Evans, C., Lentz, D. \& Roemer, M. (2009). J. Am. Chem. Soc. 131, 1251-1268.

Farrugia, L. J., Evans, C. \& Tegel, M. (2006). J. Phys. Chem. A, 110, 7952-7961.

Farrugia, L. J., Kočovský, P., Senn, H. M. \& Vyskočil, Š. (2009). Acta Cryst. B65, 757-769.

Farrugia, L. J. \& Macchi, P. (2009). J. Phys. Chem. A, 113, 1005810067.

Feixas, F., Matito, E., Poater, J. \& Solà, M. (2015). Chem. Soc. Rev. 44, 6434-6451.

Fradera, X., Austen, M. A. \& Bader, R. F. W. (1999). J. Phys. Chem. $A, \mathbf{1 0 3}, 304-314$.

Gatti, C. (2010). TOPOND98, interfaced to CRYSTAL09. CNRISTM, Milano, Italy.

Gatti, C. (2012). Struct. Bond. 147, 193-286.

Gatti, C. (2013a). Natta's Seeds Grow, From the crystallography and modelling of stereoregular polymers to the challenges of complex systems, Abstracts International Symposium on the occasion of the 50th anniversary of the award of the Nobel Prize for Chemistry to Giulio Natta and Ziegler, 21-22 November 2013, Politecnico di Milano, Italy. 
Gatti, C. (2013b). Phys. Scr. 87, 048102.

Gatti, C., Cargnoni, F. \& Bertini, L. (2003). J. Comput. Chem. 24, 422436.

Gatti, C. \& Lasi, D. (2007). Faraday Discuss. 135, 55-78.

Gatti, C. \& Macchi, P. (2012). Modern Charge Density Analysis. Dordrecht, Heidelberg, London, New York: Springer.

Gatti, C., Orlando, A. M. \& Lo Presti, L. (2015). Chem. Sci. 6, 38453852.

Gatti, C., Orlando, A. M., Monza, E. \& Lo Presti, L. (2016). Applications of Topological Methods in Molecular Chemistry, edited by R. Chauvin, C. Lepetit, B. Silvi \& E. Alikhani, Springer series Challenges and Advances in Computational Chemistry and Physics 22. New York: Springer.

Gatti, C., Saleh, G., Lo Presti, L. \& Monza, E. (2012). Sagamore meeting XVII on Charge Spin and Momentum Densities, Abstracts, 15-20 July, Daini Meisui Tei, Sapporo, Hokkaido, Japan.

Godbout, N., Salahub, D. R., Andzelm, J. \& Wimmer, E. (1992). Can. J. Chem. 70, 560-571.

Hansen, N. K. \& Coppens, P. (1978). Acta Cryst. A34, 909-921.

Hey, J., Leusser, D., Kratzert, D., Fliegl, H., Dieterich, J. M., Mata, R. A. \& Stalke, D. (2013). Phys. Chem. Chem. Phys. 15, 2060020610.

Lo Presti, L., Ellern, A., Destro, R., Soave, R. \& Lunelli, B. (2011). J. Phys. Chem. A, 115, 12695-12707.

Lo Presti, L. \& Gatti, C. (2009). Chem. Phys. Lett. 476, 308-316.

Matito, E., Duran, M. \& Solà, M. (2005). J. Chem. Phys. 122, 014109.

Matta, C. F. \& Hernández-Trujillo, J. (2003). J. Phys. Chem. A, 107, 7496-7504.

McGrady, G. S., Sirsch, P., Chatterton, N. P., Ostermann, A., Gatti, C., Altmannshofer, S., Herz, V., Eickerling, G. \& Scherer, W. (2009). Inorg. Chem. 48, 1588-1598.
Monza, E., Gatti, C., Lo Presti, L. \& Ortoleva, E. (2011). J. Phys. Chem. A, 115, 12864-12878.

Morse, P. M. \& Feshbach, H. (1981). Methods of Theoretical Physics, Part I, ch. 1. Minneapolis: Feshbach Publishing, LLC.

Oddershede, J. \& Larsen, S. (2004). J. Phys. Chem. A, 108, 10571063.

Pal, R., Mukherjee, S., Chandrasekhar, S. \& Guru Row, T. N. (2014). J. Phys. Chem. A, 118, 3479-3489.

Poater, J., Duran, M., Solà, M. \& Silvi, B. (2005). Chem. Rev. 105, 3911-3947.

Poater, J., Fradera, X., Duran, M. \& Solà, M. (2003). Chem. Eur. J. 9, 400-406.

Ponec, R. \& Gatti, C. (2009). Inorg. Chem. 48, 11024-11031.

Saleh, G. (2014). PhD thesis. Università degli Studi, Milano, Italy.

Saleh, G., Soave, R., Lo Presti, L. \& Destro, R. (2013). Chem. Eur. J. 19, 3490-3503.

Schmökel, M. S., Cenedese, S., Overgaard, J., Jørgensen, M. R. V., Chen, Y.-S., Gatti, C., Stalke, D. \& Iversen, B. B. (2012). Inorg. Chem. 51, 8607-8616.

Stewart, R. F., Bentley, J. \& Goodman, B. (1975). J. Chem. Phys. 63 , 3786-3793.

Volkov, A., Macchi, P., Farrugia, L. J., Gatti, C., Mallinson, P., Richter, T. \& Koritsanszky, T. (2006). XD2006. University at Buffalo, State University of New York, NY, USA; University of Milano, Italy; University of Glasgow, UK; CNRISTM, Milano, Italy; Middle Tennessee State University, TN, USA.

Vyskočil, S., Meca, L., Tišlerová, I., Císařová, I., Polášek, M., Harutyunyan, S. R., Belokon, Y. N., Stead, R. M. J., Farrugia, L., Lockhart, S. C., Mitchell, W. L. \& Kočovský, P. (2002). Chem. Eur. J. 8, 4633-4648. 